\title{
Subharmonic resonance and chaos in forced excitable systems
}

\author{
Hans G. Othmer, Min Xie* \\ Department of Mathematics, University of Utah, Salt Lake City,UT, 84112, USA. \\ e-mail:othmer@math.utah.edu
}

Received: 6 July 1998/Revised version: 2 October 1998

\begin{abstract}
Forced excitable systems arise in a number of biological and physiological applications and have been studied analytically and computationally by numerous authors. Existence and stability of harmonic and subharmonic solutions of a forced piecewise-linear Fitzhugh-Nagumo-like system were studied in Othmer ad Watanabe (1994) and in Xie et al. (1996). The results of those papers were for small and moderate amplitude forcing. In this paper we study the existence of subharmonic solutions of this system under large-amplitude forcing. As in the case of intermediate-amplitude forcing, bistability between $1: 1$ and $2: 1$ solutions is possible for some parameters. In the case of large-amplitude forcing, bistability between $2: 2$ and $2: 1$ solutions, which does not occur in the case of intermediate-amplitude forcing, is also possible for some parameters. We identify several new canonical return maps for a singular system, and we show that chaotic dynamics can occur in some regions of parameter space. We also prove that there is a direct transition from 2:2 phase-locking to chaos after the first period-doubling bifurcation, rather than via the infinite sequence of period doublings seen in a smooth quadratic interval map. Coexistence of chaotic dynamics and stable phase-locking can also occur.
\end{abstract}

Key words: Phase locking - Fitzhugh-Nagumo

\section{Introduction}

Excitable or oscillatory dynamics occur in many models of biological systems, including cardiac tissue (Glass et al. 1987, Chialvo 1990a,

\footnotetext{
*Supported in part by NIH grant GM 29123.
} 
Glass and Zeng 1990, Lewis and Guevara 1990), neuronal systems, and secretory systems (Wetsel et al. 1992, Sturis et al. 1991). Such systems are often subject to a periodic stimulus and the question arises as to when they can be entrained. The analysis of forced oscillators has a long history and for sufficiently small amplitude forcing it generally reduces to the study of an invertible circle map. The asymptotic dynamics in such systems are completely characterized by the rotation number. In recent years, forced excitable systems have been studied by various authors. In Alexander et al. (1989a) and Alexander et al. (1990) a theory of forced excitable systems was developed that parallels the classical theory for forced oscillators, but in these papers either the forcing had to appear in the equations for the slow variables or it had to vary slowly enough so that it could be transferred to the slow variables. Thus, the theory does not apply when the forcing varies rapidly in time and is applied to the 'fast' or 'voltage-like' variable. This type of forcing is frequently used in the experimental context (cf. Chialvo and Jalife (1987a)), and for this reason Othmer and Watanabe (1994) (hereafter referred to as I) began a study of such systems.

In I a piece-wise linear model system was introduced. The governing equations are given by

$$
\begin{aligned}
\varepsilon \frac{d v}{d t}=f(v)-w+\psi(t) & \equiv F(v, w, t), \\
\frac{d w}{d t}=v-\delta w & \equiv G(v, w) .
\end{aligned}
$$

Here $f: \mathbb{R} \rightarrow \mathbb{R}$ is the continuous, piecewise linear function defined by

$$
f(v)=\left\{\begin{aligned}
-\alpha_{1} v+\gamma_{1} & \text { for } v<v_{\ell}, \\
\alpha_{2} v-\gamma_{2} & \text { for } v_{l} \leqq v<v_{r}, \\
-\alpha_{3} v+\gamma_{3} & \text { for } v \geqq v_{r},
\end{aligned}\right.
$$

where $\alpha_{i}$ and $\gamma_{i}, i=1,2,3$, are fixed positive constants and $0<v_{\ell}<v_{r}$. Without loss of generality we assume that $\gamma_{1}=0$. The forcing function $\psi: \mathbb{R} \rightarrow \mathbb{R}$ is a piecewise constant periodic function of period $T$ defined by

$$
\psi(t)=\left\{\begin{array}{ll}
0 & \text { for } m T \leqq t<(m+\theta) T, \\
A & \text { for }(m+\theta) T \leqq t<(m+1) T,
\end{array} \quad m=0, \pm 1, \pm 2, \ldots,\right.
$$

where $A>0$ and $\theta \in[0,1]$.

Four distinct regimes were found for the amplitude of the forcing: a small-amplitude regime which produces only subthreshold responses, one intermediate-amplitude regime that corresponds to 
forcing between fixed points, and two large-amplitude regimes, one of which corresponds to forcing between a fixed point and a periodic orbit and the other to forcing between two fixed points. In I it was shown that there may be up to six distinct types of harmonic solutions, depending on the amplitude, and the dependence of the existence and stability of these solutions on the basic cycle length or period of the forcing and on the fraction of time the forcing is on was studied.

In Xie et al. (1996) (hereafter referred to as II), we studied existence of subharmonic solutions (see Sect. 2) of the singular system for an intermediate amplitude of the forcing, and we showed that there are several new phenomena that do not arise when the forcing varies slowly. We identified several canonical types of return maps and showed that there are only $n: 1$ solutions when $\theta$ is sufficiently small or sufficiently large by studying the canonical maps analytically. We identified regimes in which period-adding occurs and regimes in which the rotation number is not unique. Also we showed that the phase-locked solutions in the singular limit persist in the non-singular system.

In this paper we study subharmonic solutions for large amplitude forcing. It turns out there are no new phenomena for the case corresponding to forcing between two fixed points, so we give only a brief discussion of this case in Sect. 3. However, when the attractor is a fixed point when the forcing is off and a periodic orbit when it is on, we show that there are many phenomena similar to what was found in II, but several new phenomena as well. In Sect. 2, we repeat some of the setup given in II so as to make this paper self-contained. As in II, we study a map derived from the singular system, and in Sect. 3 we analyze subharmonic solutions for the case in which the stable attractor is a fixed point when the stimulus is off and a periodic orbit when it is on. We identify three new canonical types of return maps, and in Sect. 4 we show that chaos can occur for some parameters. In Sect. 5 we discuss the relationship of our results to those obtained by using empiricallyderived maps, as for example in Vinet et al. (1990). This connection is important in order to gain insight into complicated physiologicallyrealistic models from simplified models. We also discuss the relationship between forced excitable systems and forced oscillators. In the appendix we analyze the stability of the subharmonic solutions whose existence is shown in Sect. 3.

\section{Setup}

As we remarked in the Introduction, in I and II we studied a piecewise linear system with step function forcing. In the context of 


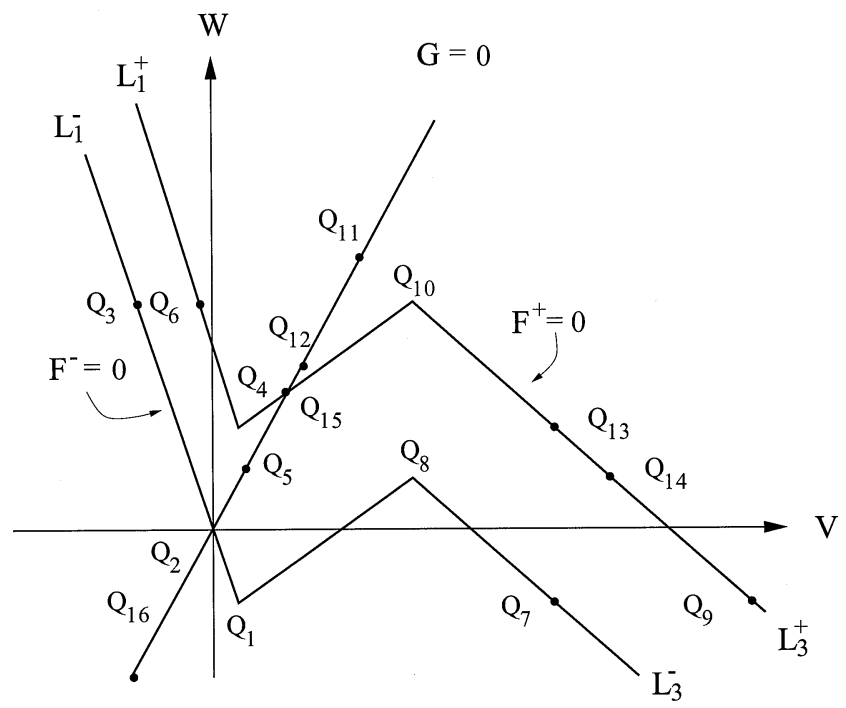

Fig. 1. A schematic of the nullclines of (1). The analytic definition of the lines is given in Table 1, and the coordination of the labeled points are given in Table 2. The second column of Table 2 gives the coordinates for the standard values of the parameters, which are $\alpha_{1}=2.0, \alpha_{2}=\alpha_{3}=1.0, \delta=0.5, \gamma_{2}=0.6$, and $\gamma_{3}=1.8$. For these parameters $A_{1}=0.4, A_{2}=0.8, A_{3}=1.8, v_{l}=0.2$, and $v_{r}=1.2$. For clarity of labeling the case $W_{4}>W_{8}$ is shown.

Table 1. The analytic definitions of the lines shown in Fig. 1

Labels

Equations

\begin{tabular}{ll}
\hline$L_{1}^{-}$ & $w=-\alpha_{1} v, v \leqq v_{l}$ \\
$L_{1}^{+}$ & $w=-\alpha_{1} v+A, v \leqq v_{\ell}$ \\
$L_{2}^{-}$ & $w=\alpha_{2} v-\gamma_{2}, v_{\ell}<v \leqq v_{r}$ \\
$L_{2}^{+}$ & $w=\alpha_{2} v-\gamma_{2}+A, v_{l}<v \leqq v_{r}$ \\
$L_{3}^{-}$ & $w=-\alpha_{3} v+\gamma_{3}, v>v_{r}$ \\
$L_{3}^{+}$ & $w=-\alpha_{3} v+\gamma_{3}+A, v>v_{r}$ \\
\hline
\end{tabular}

Fitzhugh-Nagumo-like models of excitable membranes, where $v$ represents the voltage, this forcing corresponds to injection of a depolarizing current during a fraction $(1-\theta) T$ of each cycle. Figure 1 shows the nullclines of (1), Table 1 gives the analytic definitions of the lines, and Table 2 gives the definitions of the special points shown in that figure.

The system at (1) is an autonomous system in each of the time intervals $[m T,(m+\theta) T)$ and $[(m+\theta) T,(m+1) T)$, and the solution 


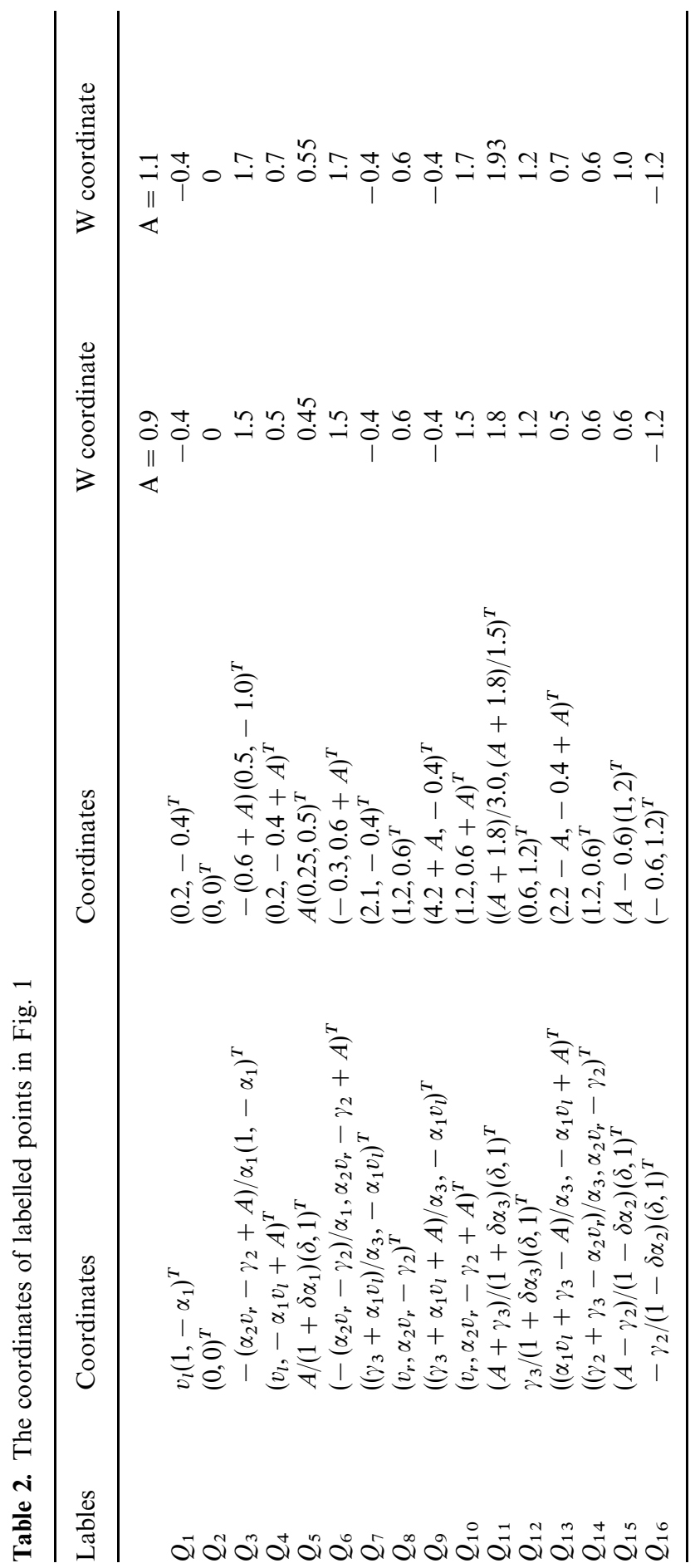


can be constructed explicitly in each of these intervals. These solutions must be pieced together to construct a solution that passes through two or more of the regions, but this cannot be done explicitly, for the times spent in the various regions satisfy transcendental equations. Thus we first study the singular limit $\varepsilon=0$, as in I and II. In the singular limit we can explicitly construct solutions valid for arbitrary time intervals because the system reduces to a linear scalar equation. As we showed in I and II, in the singular limit $\varepsilon=0$ (1) reduces to

$$
\begin{array}{ll}
\frac{d w}{d t}=-\lambda_{i}\left(w-w_{i}^{*}\right) & \text { for }(v, w) \in \mathbf{L}_{i}^{-}, \\
\frac{d w}{d t}=-\lambda_{i}\left(w-\left(w_{i}^{*}+A_{i}^{*}\right)\right) & \text { for }(v, w) \in \mathbf{L}_{i}^{+}, \quad i=1,3 .
\end{array}
$$

where

$$
w_{i}^{*} \equiv \frac{\gamma_{i}}{1+\delta \alpha_{i}} \quad \text { and } \quad A_{i}^{*} \equiv \frac{A}{1+\delta \alpha_{i}} .
$$

The $w_{i}^{*}$ (resp., $\left.w_{i}^{*}+A_{i}^{*}\right)$ are the $w$ coordinates of the rest points (resp., virtual rest points, i.e., the intersection of the extension of $\mathbf{L}_{i}^{ \pm}$ with $G=0$ ), and from Fig. 1 and Table 2 one sees that $w_{1}^{*}=0$, $w_{1}^{*}+A_{1}^{*}=W_{5}, \quad w_{3}^{*}=W_{12}$, and $w_{3}^{*}+A_{3}^{*}=W_{11}$. We denote by $\phi\left(t, P_{0}\right) \equiv\left(\mathscr{V}\left(w\left(t, P_{0}\right), t\right), w\left(t, P_{0}\right)\right)$ the solution of these equations and $F(v, w, t)=0$, with the initial value $\phi\left(0, P_{0}\right)=P_{0} \equiv\left(v_{0}, w_{0}\right)$.

Since solutions are constrained to the lines $\mathbf{L}_{i}^{ \pm}$when $\varepsilon=0$, we assign the symbols, $0,1,2$ and 3 to the segments $\overline{Q_{1} Q_{3}}, \overline{Q_{4} Q_{6}}, \overline{Q_{7} Q_{8}}$, and $\overline{Q_{9} Q_{10}}$, respectively, and we describe solutions as follows. To each solution we attach a semi-infinite symbol sequence $\mathcal{I} \equiv$ $\left(a_{0}, a_{1}, a_{2}, \ldots\right)$, where $a_{i} \in\{0,1,2,3\}$ and $a_{0}$ is either 0 or 2 . The solution $\mathcal{I}_{n}$ for $n$ cycles of the forcing is a subsequence of $\mathcal{I}$ (see I and II for details)

Given a sequence $\mathcal{I}$, we define the firing number or rotation number $\rho$ for that sequence as the asymptotic ratio of the number of 3's, with certain 3's excluded, to the number of forcing cycles. More precisely, let $n_{i}$ be the number of $a_{i}$ 's in $\mathcal{I}_{n}$, and let $n_{23}$ be the number of ordered pairs $(2,3)$ in $\mathcal{I}_{n}$; then $n_{23}$ represents the number of jumps from $L_{3}^{-}$to $L_{3}^{+}$in $\mathcal{I}_{n}$. We define the firing or rotation number as

$$
\rho=\limsup _{n \rightarrow \infty} \frac{n_{3}-n_{23}}{n}
$$

whenever this limit exists. According to this definition, any solution that visits the segment $\overline{Q_{9} Q_{10}}$ only finitely many times has firing 
number zero. We refer to such solutions as subthreshold solutions and label them 1:0. Periodic solutions with firing number equal to 1 are called harmonic solution, those with a firing number less than 1 are called subharmonic solutions, and those with firing number greater than 1 are called superharmonic solutions. Subharmonics can be ruled out if the product $\theta T$ is large enough, i.e. if the forcing is off sufficiently long for fixed $T$, as the following shows.

Proposition 1 (Xie et al., 1996). If $W_{4}>W_{8}$ and

$$
\theta T>\max \left\{T_{i}^{-}\left(W_{3}, W_{4}\right), T_{3}^{-}\left(0, W_{8}\right)\right\}
$$

or if $W_{4}<W_{8}$ and

$$
\theta T>\max \left\{T_{1}^{-}\left(W_{3}, W_{4}\right), T_{3}^{-}\left(0, W_{8}\right)+T_{1}^{-}\left(W_{8}, W_{4}\right)\right\}
$$

then there are no subharmonic solutions.

Superharmonic solutions are not treated here; our purpose is to analyze the subharmonic solutions as a function of the parameters $\theta$, $T$ and $A$, and the proposition shows that $\theta T$ must be small enough. To this end we identify four distinct cases for the location of the 'rest point', and these are characterized by the forcing amplitude as follows $A>A_{3}$

$$
\begin{array}{rll}
\text { I. } & Q_{5} \in \mathbf{L}_{1}^{+} & \text {for } 0<A \leqq A_{1}, \\
\text { II. } & Q_{5} \in \mathbf{L}_{1}^{+} & \text {for } A_{1}<A \leqq A_{2}, \\
\text { III. } & Q_{15} \in \mathbf{L}_{2}^{+} & \text {for } A_{2}<A \leqq A_{3}, \\
\text { IV. } & Q_{11} \in \mathbf{L}_{3}^{+} & \text {for } A>A_{3},
\end{array}
$$

where

$$
A_{1}=\alpha_{1} v_{l}, \quad A_{2}=\frac{\left(1+\delta \alpha_{1}\right) v_{\ell}}{\delta}, \quad A_{3}=\gamma_{2}+\frac{\left(1-\delta \alpha_{2}\right) v_{r}}{\delta} .
$$

In cases I and II the 'rest point' is on $\mathbf{L}_{1}^{+}$while the forcing is on, while in Case III it is on $\mathbf{L}_{2}^{+}$. In Case IV the stable attractor is a rest point on $\mathbf{L}_{3}^{+}$when the forcing is on and a rest point on $\mathbf{L}_{1}^{-}$when it is off. In Case III the solution jumps from $\mathbf{L}_{1}^{+}$to $\mathbf{L}_{3}^{+}$at $Q_{4}$, and from $\mathbf{L}_{3}^{+}$to $\mathbf{L}_{1}^{+}$at $Q_{10}$ periodically as long as the forcing remains on, and the time $T(A)$ required for each circuit is

$$
T(A)=\ln \left[\left(\frac{W_{10}-W_{5}}{W_{4}-W_{5}}\right)^{1 / \lambda_{1}}\left(\frac{W_{4}-W_{11}}{W_{10}-W_{11}}\right)^{1 / \lambda_{3}}\right] .
$$


Let $T_{i}^{ \pm}\left(w_{0}, w\right)$ denote the time to reach $w$ from $w_{0}$ while remaining on $\mathbf{L}_{i}^{ \pm}$. Then

$$
T_{i}^{-}\left(w_{0}, w\right)=\frac{1}{\lambda_{i}} \ln \left[\frac{w_{0}-w_{i}^{*}}{w-w_{i}^{*}}\right]
$$

and

$$
T_{i}^{+}\left(w_{0}, w\right)=\frac{1}{\lambda_{i}} \ln \left[\frac{w_{0}-\left(w_{i}^{*}+A\right)}{w-\left(w_{i}^{*}+A\right)}\right] .
$$

As in I and II, we shall use a return map to study the existence of periodic solutions, and here we indicate how it is constructed for the reader's convenience. We map the $w$-coordinate into the unit interval via the transformation

$$
x= \begin{cases}\frac{w}{W_{8}+W_{10}} & \text { if }(v, w) \in L_{1}^{-} \text {and } w \geqq 0, \\ 1-\frac{w}{W_{8}+W_{10}} & \text { if }(v, w) \in L_{3}^{-} \text {and } w \geqq 0 .\end{cases}
$$

In effect this mapping takes the segment of $L_{3}^{-}$lying between $Q_{8}$ and the $v$ axis and appends it to $L_{1}^{-}$(truncated above $W_{10}$ ) and rescales the result. Thus the segment of $L_{1}^{-}$lying between the origin and the intersection of the horizontal line with $w$-coordinate $W_{4}$ is mapped into the interval $\left[0, x_{4}\right]$, and so on. By a period one or return map $\mathscr{F}$ we mean a map from the unit interval to itself that maps a point into its image after time T. Case III has to be split into two subcases corresponding to $W_{4}<W_{8}$, and $W_{4}>W_{8}$, and maps for these cases are shown in Fig. 2. Since $W_{4}=W_{8}$ when $A=1.0$, we choose $A=0.9$ and $A=1.1$, as representatives for these cases. These figures will be referred to again later, but we show them here to explain some of the characteristics of the return maps.

To understand these maps, consider first the interval $\left(0, x_{4} e^{\lambda_{1} \theta T}\right]$. Points in this interval map to the interval $\left(0, x_{4}\right]$ while the forcing is off, and then jump to $\mathbf{L}_{3}^{+}$when it turns on. For the standard parameters given in the legend to Fig. 1, one finds that the interval $\left(0, x_{4} e^{\lambda_{1} \theta T}\right]$ is mapped into $\left(1-x_{8}, x_{8}\right)$, and in either case the orientation is preserved. The second characteristic interval is $\left(x_{4} e^{\lambda_{1} \theta T}, x_{8}\right)$, and this is mapped into either $\left(x_{4}, 1\right)$ (in Fig. 2(a)) or $\left(1-x_{8}, x_{8}\right)$ (in Fig. 2(b)), and in both cases there are intervals whose orientation is reversed and intervals whose orientation is preserved. The first discontinuity in (a) arises when the flow fails to reach $Q_{14}$ on $L_{3}^{+}$while the forcing is on, and therefore is mapped into $\left(x_{8}, 1\right)$. For still larger $x$ the flow fails to reach 

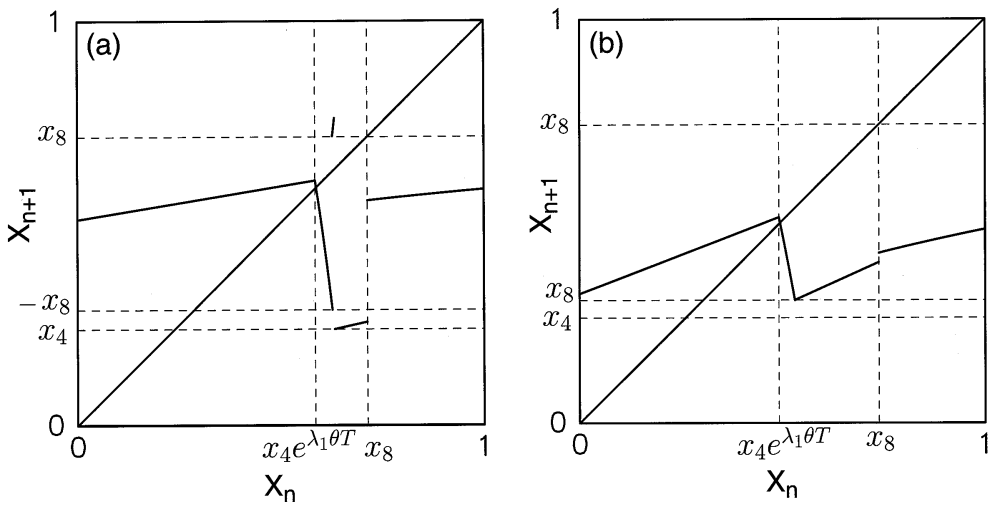

Fig. 2. The computed return map for (a) $(A, T, \theta)=(0.9,1.5,0.6)$, and (b) $(A, T, \theta)=$ $(1.1,0.8,0.6)$.
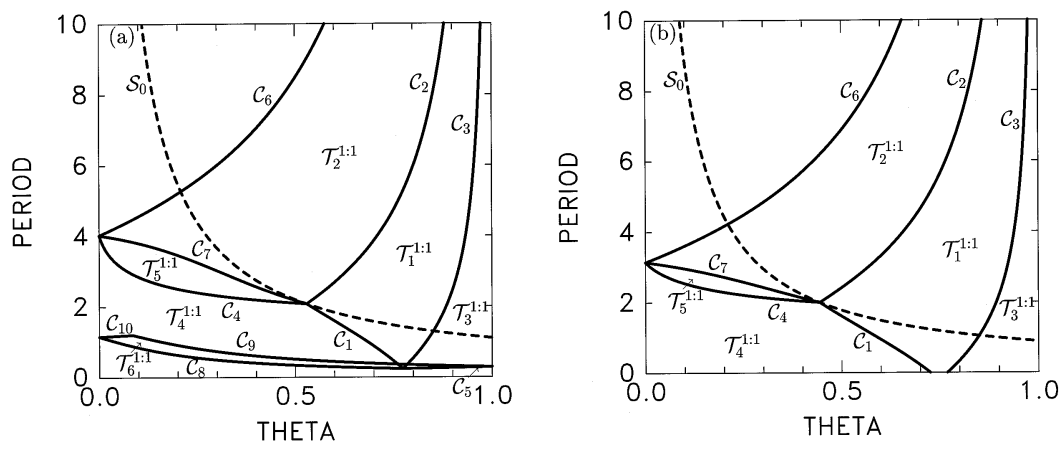

Fig. 3. A diagram of the phase-locking regions for $1: 1$ solutions (a) for $A=0.9$ and (b) for $A=1.1$ (corresponding to $W_{4}<W_{8}$ and $W_{4}>W_{8}$, respectively). There are no subharmonic solutions in the region above the dashed line labeled $\mathcal{S}_{0}$. The label $\mathcal{T}_{i}^{1: 1}$ denotes the region in which $1: 1$ solutions of type $i$ exist and $\mathcal{C}_{j}$ denotes a boundary of the regions.

$Q_{4}$, which accounts for the second discontinuity. As one sees in (b), when $W_{4}>W_{8}$ neither of these discontinuities arise for this choice of $(A, T, \theta)$. In fact, in (b) the interval $\left(0, x_{8}\right)$ is invariant and attracting, and the return map is continuous and piecewise smooth in this interval. As we will see later, the dynamics in these two cases can be quite different.

In Fig. 3 we show the locking regions for $1: 1$ solutions for $A \in\left(A_{2}, A_{3}\right)$ (Case III), as well as the curve $\mathcal{S}_{0}$ defined by the conditions in Proposition $1^{1}$. As $A \rightarrow A_{2}$ (the lower limit for Case III), $\mathcal{C}_{6}$, one of the boundaries of the region $\mathcal{T}_{2}$, tends to the axis $\theta=0$ and the regions in which type 4-6 solutions exist vanish, whereas when $A \rightarrow A_{3}$

\footnotetext{
${ }^{1}$ See Figs. 6 and 10 in I for definitions of the various 1 : 1solutions.
} 
the curves $\mathcal{C}_{2}, \mathcal{C}_{4}, \mathcal{C}_{6}$, and $\mathcal{C}_{7}$ tend to the left-upper corner, the regions of solutions of types 2,5 , and 6 disappear, and there are only solutions of types 1,3 and 4 in the $(\theta, T)$ space. Note that for Case II (i.e., $\left.A \in\left(A_{1}, A_{2}\right)\right)$, only $A=0.6$ was analyzed in I and II, but the results are quite similar throughout a region.

The analysis of subharmonic resonance is more complicated in Case III than that in Case II, and in this paper we restrict attention to this case. In the following section we first give a brief discussion of the case $A>A_{3}$ and then restrict attention to Case III. We analyze the regions of existence of subharmonic solutions, and in particular, we determine how the locking regions or resonance zones for subharmonic solutions are arranged in the parameter space $(\theta, T)$. That is, we determine what types of sequences of transitions between different rotation numbers are observed along paths in $(\theta, T)$-space.

\section{Existence of subharmonic solutions}

\subsection{Resonance in Case IV}

When the amplitude of the forcing $A$ is greater than $A_{3}$, the stable attractor is a fixed point on the right branch of the nullcline $\dot{v}=0$ when the forcing is on. In this case solutions of the singular system can never reach $W_{10}$, and hence there are no solutions of type 2 or 5 . There are two subcases determined by whether the $w$ coordinate of the fixed point is greater or less than $W_{4}$ (i.e., the fixed point is above or below $Q_{4}$ ). In the former subcase there are solutions of types 1 and 4 for small $\theta$; harmonic solutions if the period $T$ is large and subharmonic solutions if $T$ is small. For large $\theta$ (i.e., short stimuli) there are only type 3 solutions. These are harmonic solutions if $W_{4}>W_{8}$, but otherwise there may be subharmonic solutions of type 3 .

In the second subcase the $w$ coordinate of the fixed point is less than $W_{4}$ (i.e., the fixed point is below $Q_{4}$ ), and to obtain this we must have $W_{4}>W_{8}$. Thus, there are no solutions of types 4 and 6 , and there are only harmonic solutions of type 1 and type 3 . We summarize the above discussion in the following proposition.

Proposition 2. Suppose that $A>A_{3}$; then the following properties hold.

(a) There are no solutions of types 2 and 5 .

(b) For large $\theta$ (short stimuli), if the fixed point is above $Q_{4}$, then there are only type 3 solutions. In particular, there are only harmonic solutions if $W_{4}>W_{8}$. 
(c) For small $\theta$ (long stimuli), if the fixed point is above $Q_{4}$, then there may be both harmonic and subharmonic solutions.

(d) If the fixed point is below $Q_{4}$, then there are no solutions of types 4 and 6, and there are only harmonic solutions of types 1 and 3.

Remark 1. Property (b) of the Proposition, is also true for $A \in\left(A_{2}, A_{3}\right)$.

\subsection{Numerical results on resonance in Case III}

To see how the resonance zones corresponding to different subharmonics are arranged in the $(\theta, T)$ plane when the attractor alternates between a fixed point and a periodic orbit, we have computed the rotation number numerically for various combination of the parameters. The results shown in Fig. 4 are for a fixed $A$ corresponding to each of the two subcases in Case III, namely, $A=0.9\left(W_{4}<W_{8}\right)$ and $A=1.1\left(W_{4}>W_{8}\right)$, and various values of $\theta$. The rotation number is apparently non-monotonic in the period and is multiple-valued for some values of the period. This implies that multistability may occur in
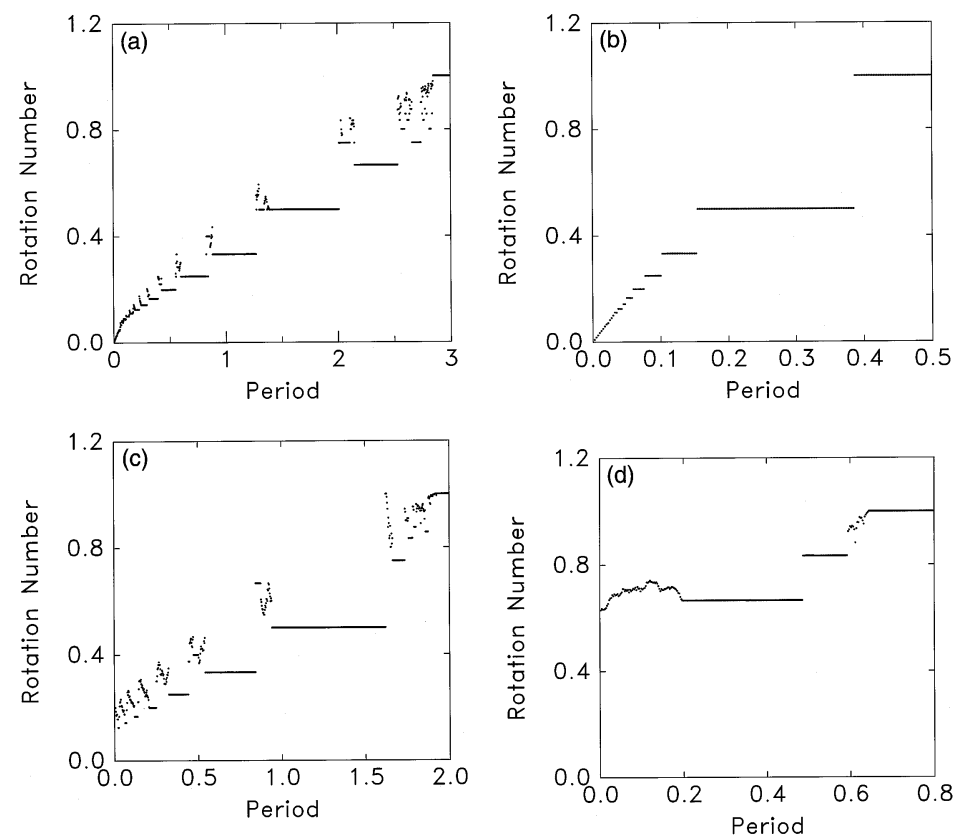

Fig. 4. The rotation number as a function of the period for several combinations of $A$ and $\theta . \quad$ (a) $(A, \theta)=(0.9,0.1), \quad$ (b) $(A, \theta)=(0.9,0.95), \quad(\mathbf{c})(A, \theta)=(1.1,0.1)$, (d) $(A, \theta)=(1.1,0.6)$. 
the system for Case III. There are two types of multistability: one in which there are distinct stable solutions with the same rotation number, and one in which there are distinct stable solutions having different rotation numbers. In the following paragraphs, we examine the two types of multistability in several examples.

In Fig. 4 one cannot distinguish between solutions for which $\rho=p / q$, and those for which $\rho=k p / k q$ for $k>1$ (the first kind of multistability) but the latter case occurs frequently, particularly for $\theta$ close to 1 . Previously we showed that this happens for $A \in\left(A_{1}, A_{2}\right)$, where there are solutions with $k=1,2$, and 4 . Here not only may solutions of different $k$ coexist, but also solutions of different $k$ may coexist with solutions of different rotation numbers, as is shown in Fig. 5. All three solutions shown there are stable. We show a $7: 1$ solution with rotation number $1 / 7$ in (a) and (b), an $18: 3$ solution with rotation number $1 / 6$ in (c) and (d), and a $24: 4$ solution with rotation number $1 / 6$ in (e) and (f).

When there is coexistence of solutions of different rotation number one can ask what the nature of the transitions between them is as a parameter in the equations is varied. In II we showed that a direct transition from a $2: 1$ to a $1: 1$ solution is possible, without passing through solutions with irrational rotation number, as $T$ or $\theta$ is varied. In fact, one observes hysteresis between these solutions along suitable paths in $A-\theta$ space. The $2: 1$ to $1: 1$ (and reverse) transition occurs here as well, but only for type 3 solutions. In addition there is coexistence between stable 2:2 solutions and stable 2:1 solutions, an example of which is shown in Fig. 6. This type of bistability was first found in maps by Guevara et al. (1984) and later found by Lewis and Guevara (1990). Bistability between two stable solutions with different rotation numbers occurs in many other dynamical systems as well (Aronson et al. 1986, Boyland 1986, Rinzel and Troy 1983).

In Fig. 6(a) we show the second iterate of the return map for $(A, T, \theta)=(0.9,0.3,0.9)$ where one sees that there are three fixed points. The central one corresponds to a one-point stable orbit and the corresponding 1:1 solution is shown in Fig. 6(b). The other two correspond to a two-point stable orbit, and the corresponding $2: 1$ solution is shown in Fig. 6(c). From the return map in (a), one can see that the domain of attraction of the $1: 1$ solution is near the central fixed point and the domain of attraction of the $2: 1$ solution is near 1 and 0 . Comparing the $1: 1$ solution in (b) with the $2: 1$ solution in (c), one can see that the recovery time of the $1: 1$ solution is longer than that of the $2: 1$ solution. Thus, when the first stimulus turns on the phase point is at a point below $Q_{4}$ and a spike occurs, and when the second stimulus turns on, the system fires again when it is on the orbit 

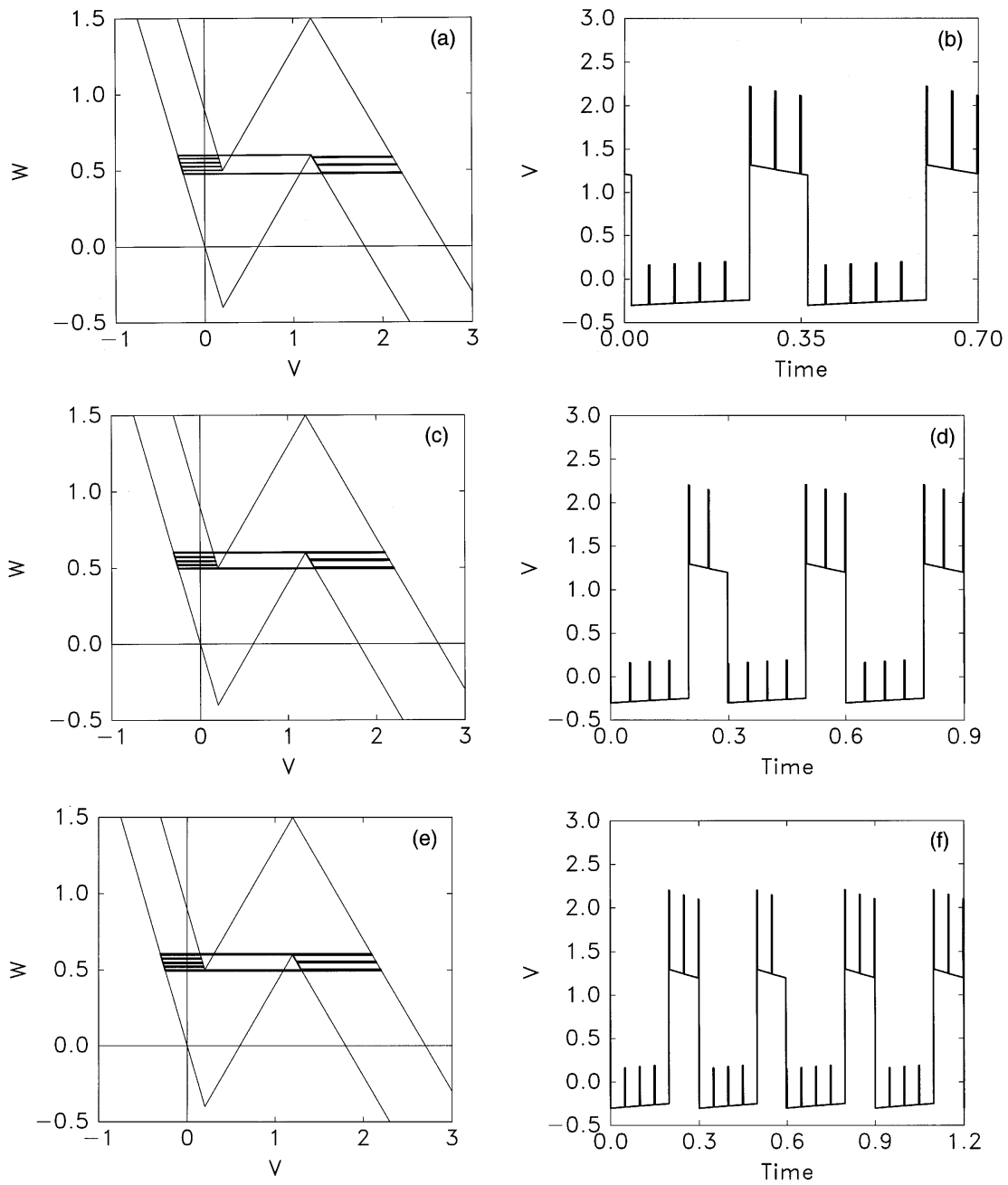

Fig. 5. The phase plane and v-component of a stable periodic solution of rotation number $\rho=1 / 7$ and two stable periodic solutions of rotation number $\rho=1 / 6$ that coexist at $(A, T, \theta)=(0.9,0.05,0.95)$. In (a) we show the phase plane and in (b) the time trace of $v$ for the 7:1 solution, in (c) and (d) we show the corresponding plots for the $18: 3$ solution, and in (e) and (f) we show the corresponding plots for the $24: 4$ solution. In each of these the transitions from $L_{3}^{-}$to $L_{3}^{+}$are not counted as firings.

corresponding to the $1: 1$ solution, but it does not fire in response to the second stimulus on the orbit for the $2: 1$ solution.

In Fig. 6(d) we shown the second iterate of the return map for $(A, T, \theta)=(0.9,2.0,0.3)$. Here there are five fixed points which belong to two distinct two-point orbits and one one-point orbit. If we order the five points by their $x$-coordinates from smallest to largest, the 

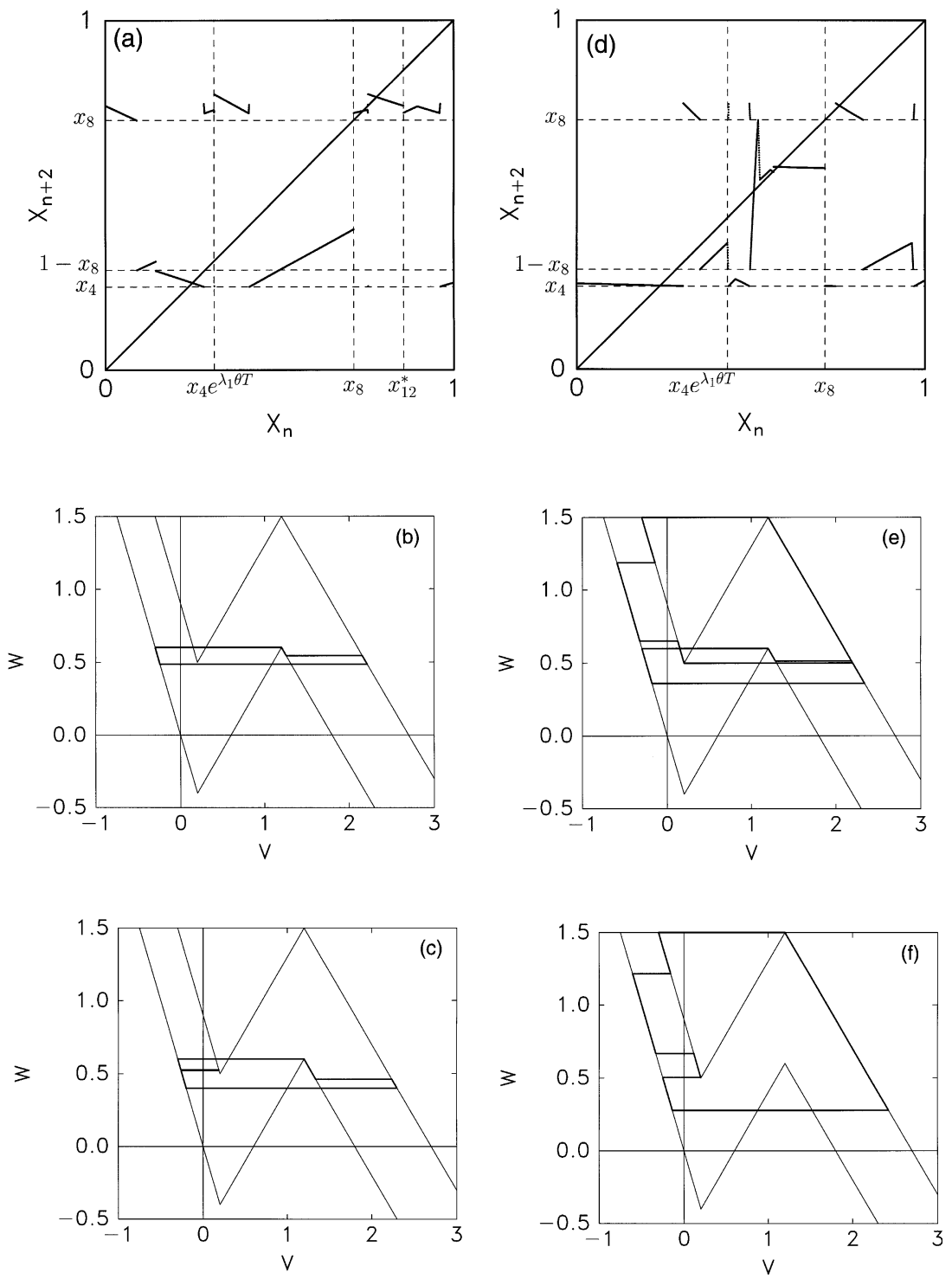

Fig. 6. The return maps and phase planes for two examples of parameter values that lead to bistability of type 3 periodic solutions. In (a)-(c) $(A, T, \theta)=(0.9,0.3,0.9)$, and in (d)-(f) $(A, T, \theta)=(0.9,2.0,0.3)$. (a) The second iterate of the return map, (b) the phase plane for the $1: 1$ solution in (a), (c) the phase plane for the $2: 1$ solution in (a); (d) the second iterate of the return map at $(A, T, \theta)=(0.9,2.0,0.3),(\mathbf{e})$ the phase plane for the $2: 2$ solution, (f) the phase plane for the $2: 1$ solution.

second point corresponds to a Type $41: 1$ unstable solution, the first and fourth points correspond to the $2: 1$ solution shown in (f), and the third and fifth points correspond to the $2: 2$ solution shown in (e). The 
flow corresponding to the return map in (d) has a large fraction of period stimulated. From Fig. 6(e) and (f), one can see that the first stimulus is turned on at a lower point for the $2: 1$ solution than for the $2: 2$ solution. The time spent on $L_{3}^{+}$for the $2: 1$ solution is longer than for the $2: 2$ solution. When the second stimulus is on, the time spent on $L_{1}^{+}$for the $2: 1$ solution is longer than for the $2: 2$ solution and hence there is no firing corresponding to the second stimulus for the $2: 1$ solution and there is a firing with short time spent on $L_{3}^{+}$for the $2: 1$ solution.

The above discussion indicates that multistability can occur in certain parameter regimes. Moreover, there is further fine structure in the locking regions. We have computed the locking regions for $2: 1$ solutions in the $\theta-T$ plane by the method used in Othmer and Watanabe (1994) (i.e., we derive equations for the fixed points and numerically compute the boundaries of the regions for each type of $2: 1$ solution). Just as there are six harmonic solutions in Case III (cf. Othmer and Watanabe (1994)), so also there are up to six types of $2: 1$ solutions, which are shown in Fig. 7. A type 1 solution (cf. (a)) spends time on $L_{3}^{+}$while the first stimulus is on at a point below $Q_{4}$ and on $L_{1}^{-}$ while it is off. In contrast, a type 4 solution (cf. (d)) spends time, first on $L_{1}^{+}$and then on $L_{3}^{+}$while the first stimulus is on at a point below $Q_{4}$, and on $L_{1}^{-}$while it s off. A type 2 or type 5 solution has a large fraction of period stimulated. A type 2 solution (cf. (b)) first spends time, on $L_{3}^{+}$ and then on $L_{1}^{+}$while the first stimulus is on at a point below $Q_{4}$, and on $L_{1}^{-}$while it is off. A type 5 solution first spends time, on $L_{1}^{+}$, and then on $L_{3}^{+}$, and then on $L_{1}^{+}$again while the first stimulus is on at a point below $Q_{4}$, and on $L_{1}^{-}$while it is off. However, a type 3 or a type 6 solution has a short fraction of period stimulated. A type 3 solution spends time on $L_{3}^{+}$while the first stimulus is on at a point below $Q_{4}$, and first on $L_{3}^{-}$and then on $L_{1}^{-}$while it is off. In contrast, a type 6 solution spends time, first on $L_{1}^{+}$and then on $L_{3}^{+}$while the first stimulus is on, and first on $L_{3}^{-}$and then on $L_{1}^{-}$while it is off. All types of solutions remains on $L_{1}^{+}$while the second stimulus is on, and on $L_{1}^{-}$ while it is off.

The locking regions for $1: 1$ and $2: 1$ solutions for $A=0.9$ $\left(W_{4}<W_{8}\right)$ and $A=1.1\left(W_{4}>W_{8}\right)$ in the $\theta-T$ plane are shown in Fig. 8. If $W_{4}<W_{8}$ there are large locking regions for $1: 1$ and $2: 1$ solutions of all types as in Case II, and small $1: 1$ and $2: 1$ overlap regions for type 3, but unlike Case II, there is no $1: 1$ and $2: 1$ overlap region for type 2. Moreover, if $W_{4}>W_{8}$, there are not type 3 and type $62: 1$ solutions, hence no bistability of $1: 1$ and $2: 1$ solutions for type 3 solutions. Similarly, there is no $1: 1$ and $2: 1$ overlap region for type 2 solutions. To understand why there is no overlap, note that 

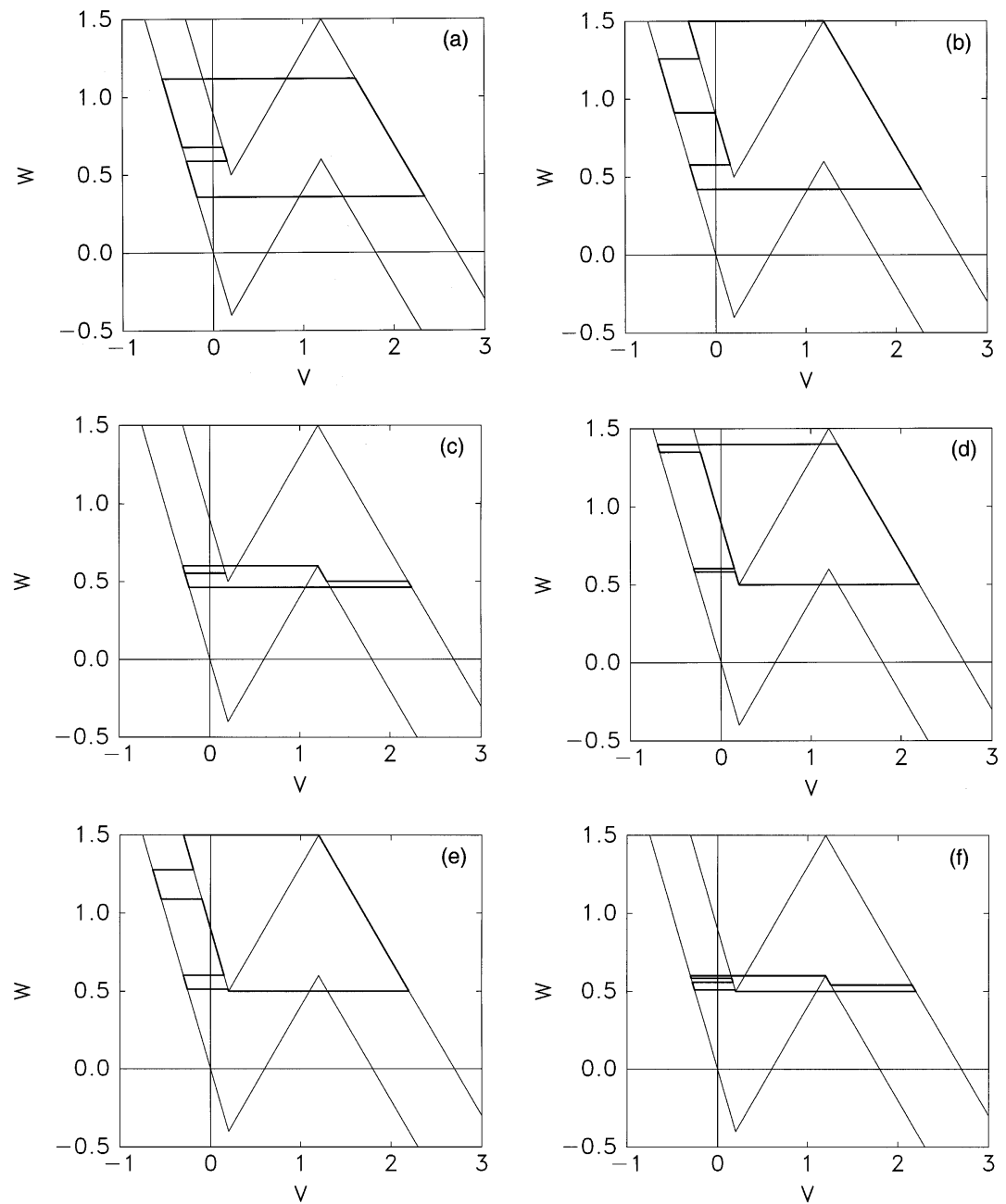

Fig. 7. The six distinct types of $2: 1$ solutions. (a) a type $12: 1$ solution at $(A, T, \theta)=(0.9,1.0,0.5),(\mathbf{b})$ a type $22: 1$ solution at $(A, T, \theta)=(0.9,1.6,0.2)$, (c) a type $32: 1$ solution at $(A, T, \theta)=(0.9,0.2,0.9)$, (d) a type $42: 1$ solution at $(A, T, \theta)=(0.9,1.8,0.02),(\mathbf{e})$ a type $52: 1$ solution at $(A, T, \theta)=(0.9,1.6,0.1),(\mathbf{f})$ a type $32: 1$ solution at $(A, T, \theta)=(0.9,0.3,0.3)$.

in Case II the attractor is a fixed point on $L_{1}^{+}$when the forcing is on. Thus, near the boundary of the type 2 harmonic locking region, the flow from an initial point on $L_{1}^{-}$below and close to $Q_{4}$ in $w$-component, jumps to $L_{3}^{+}$when the forcing turns on, and this produces the $1: 1$ solution. On the other hand, if the $w$-component of the initial point on $L_{1}^{-}$is large enough, the flow will jump to $L_{1}^{+}$when the forcing turns on, and move upward along $L_{1}^{+}$towards the attractor. Then, when the 

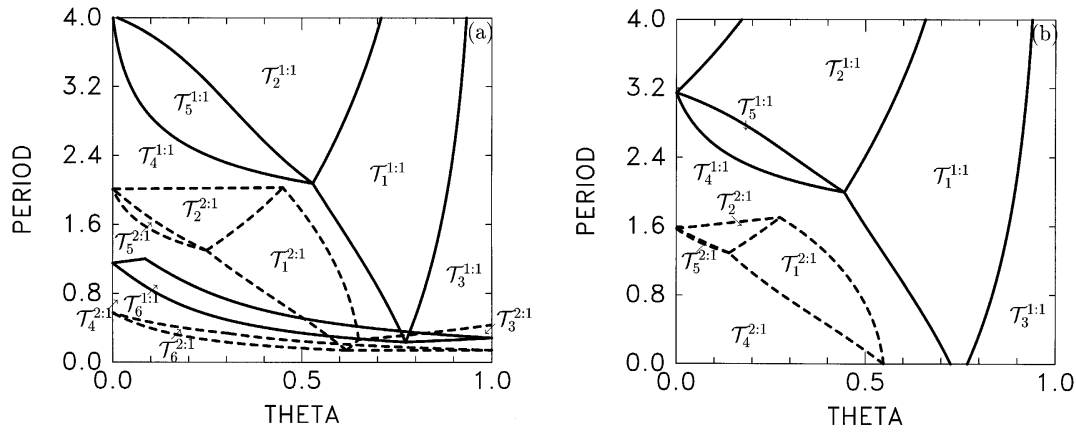

Fig. 8. Locking regions for $1: 1$ and $2: 1$ solutions for (a) $\mathrm{A}=0.9$ and (b) $\mathrm{A}=1.1$. The label $\mathcal{T}_{i}^{q: p}$ denotes the region in which $q: p$ solutions of type $i$ exist. For $1: 1$ solutions the regions are as shown in Fig. 3.

second stimulus is given, the flow from a point on $L_{1}^{-}$below and close to $Q_{4}$ in $w$-component jumps to $L_{3}^{+}$, and this produces the $2: 1$ solution. As a result, bistability between $1: 1$ and $2: 1$ solutions is possible in the small region close to the boundary of the locking region for type 2 harmonics. However this is not possible in Case III, for now the attractor is a periodic orbit when the forcing is on. Thus, near the boundary of the type 2 harmonic locking region, the flow from a point on $L_{1}^{-}$below and close to $Q_{4}$ in $w$-component jumps directly to $L_{3}^{+}$. However, when the initial point has a large enough $w$-component on $L_{1}^{-}$, the solution will jump to $L_{1}^{+}$, flow down towards $Q_{4}$, and then jump to $L_{3}^{+}$or return to $L_{1}^{-}$. In other words, either the solution jumps directly to $L_{3}^{+}$or reaches it via $L_{1}^{+}$, in which case it is a $1: 1$ solution, or it fails to reach $Q_{4}$ the first cycle but does so in the second cycle, in which case it is a $2: 1$ solution, but the two cases can not occur for the same parameters. It is also possible for the system to have a type $41: 1$ solution in this region, and thus for some $T$ there bistability between two different types of harmonic solutions. However, the type 4 solutions are unstable where they coexist with $2: 1$ solutions, and therefore there is no bistability between $1: 1$ and $2: 1$ solutions.

\subsection{Canonical maps in Case III}

Given that the 1:1 and 2:1 resonance zones do not overlap for type 1 and 2 solutions, we can ask what the dynamics are in the region between these zones. We have not computed the locking regions for other than the 2:1 subharmonic solutions, but we can gain some qualitative insights into the different subharmonic phase-locking patterns by studying representatives return maps that arise for parameter 

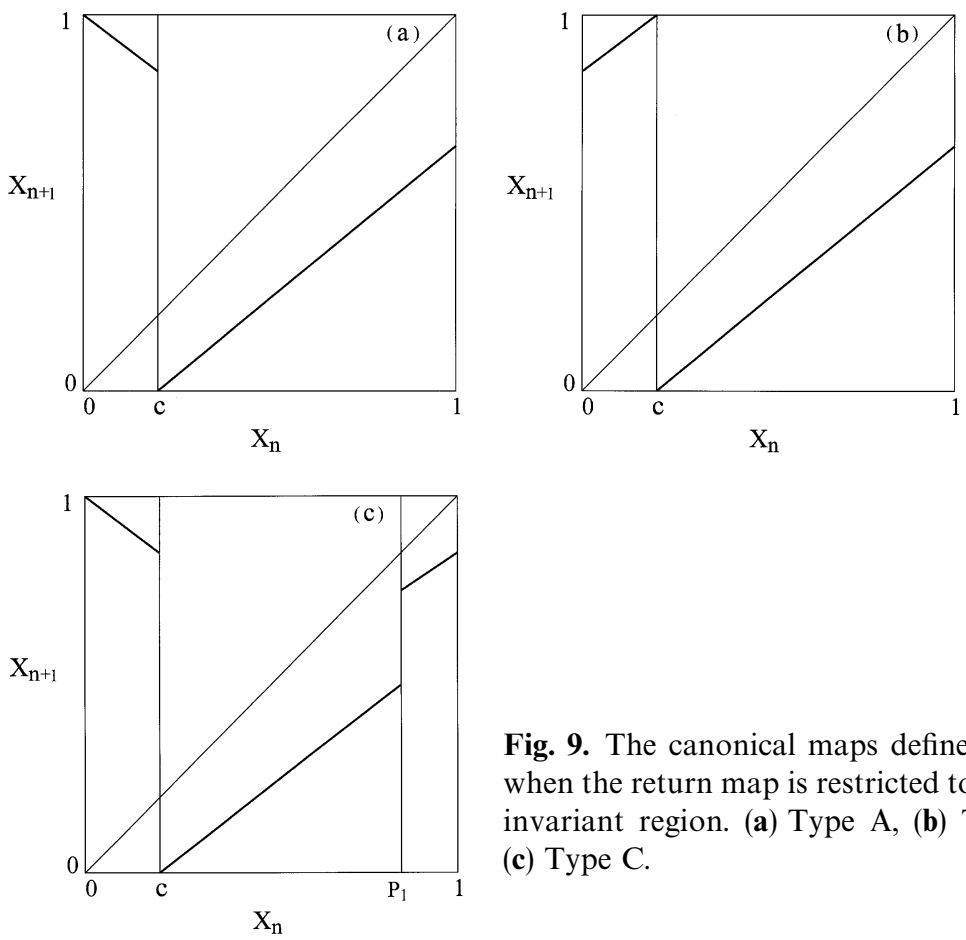

Fig. 9. The canonical maps defined in Case II when the return map is restricted to its maximal invariant region. (a) Type A, (b) Type B, and (c) Type C.

values in the $2: 1$ locking regions and in the region between $1: 1$ locking and $2: 1$ locking.

In Case II we identified three canonical forms of the return maps restricted to their minimal invariant regions and for later reference they are shown in Fig. 9. To determine what new types of maps may arise in Case III, we compute the return maps for representative parameter values. The numerically-computed graphs of the return maps for $A \in\left(A_{2}, A_{3}\right)$ and various values of $(\theta, T)$ are shown in Figs. 2 and 10. These return maps can be more complicated than those in Case II, but there are some similarities between the cases. For instance, we find that the regions in parameter space in which the return map restricted to its minimal invariant set is one of type $\mathrm{A}, \mathrm{B}$, or $\mathrm{C}$ cover most of the regions in which $2: 1$ solutions of types 1,2 , and 3 exist. In particular, most $2: 1$ solutions of types 2 and 1 have type A and B maps, respectively, and most type $31: 1$ and $2: 1$ solutions have type $\mathrm{C}$ maps if $W_{4}<W_{8}$. (If $W_{4}>W_{8}$, there are no subharmonic solutions of type 3.) A complete analytical characterization of the dynamics for Types $\mathrm{A}$ and $\mathrm{C}$ maps is given in II, and it is shown that the rotation number for Type B maps is a Cantor function along certain curves in $\Theta-T$ space. Thus much of the dynamical behavior in the $2: 1$ locking regions can be understood on the basis of the results given in II for these three types of canonical 

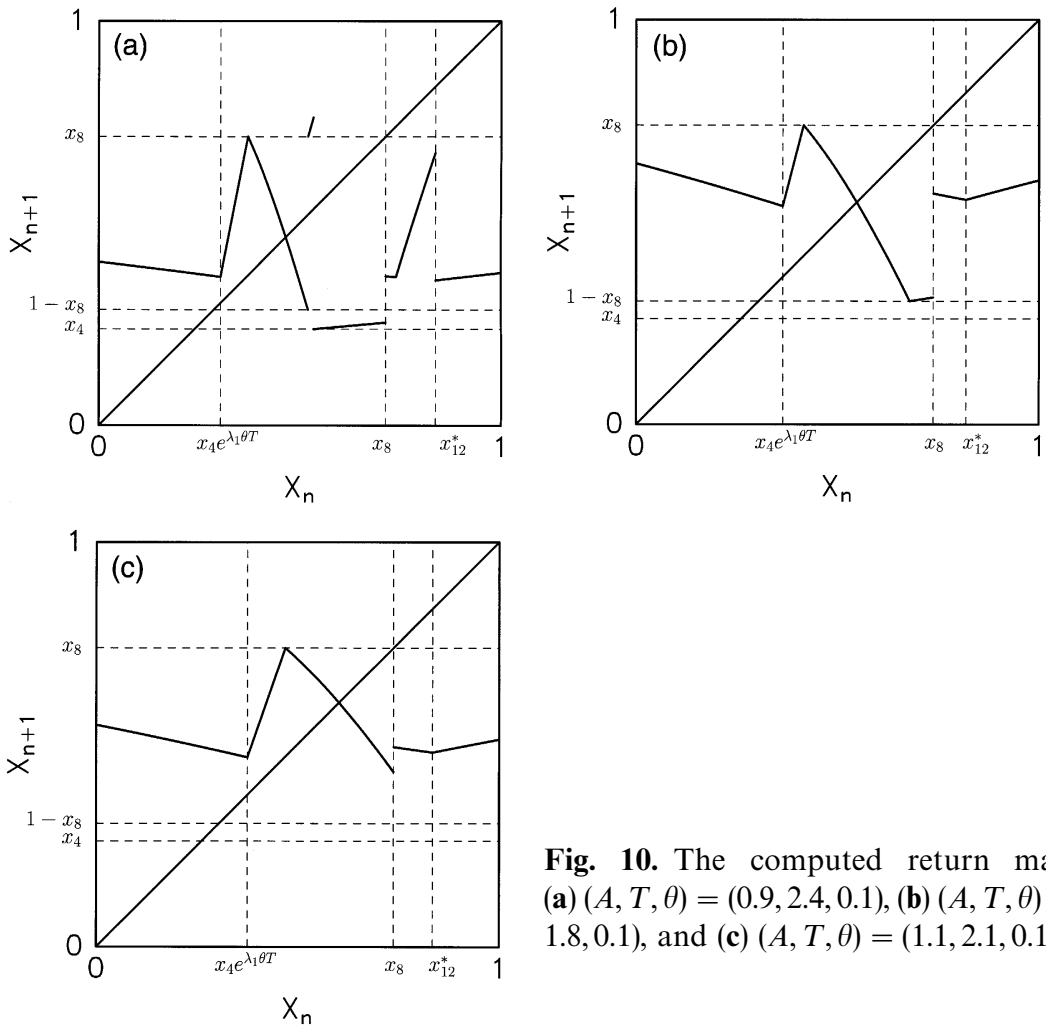

Fig. 10. The computed return map for (a) $(A, T, \theta)=(0.9,2.4,0.1)$, (b) $(A, T, \theta)=(1.1$, $1.8,0.1)$, and (c) $(A, T, \theta)=(1.1,2.1,0.1)$.

maps. When the period $T$ is small the return map is generally more complicated, as in Case II (cf. Xie et al. 1996)).

However, different types of maps can arise when the parameters lie in the region between the 2:1 locking regions and the harmonic locking regions, as is shown in Figs. 2 and 10. There Figs. 2(a) and 10(a) correspond to $W_{4}<W_{8}$, and Figs. 2(b) and 10(b) and 10(c) correspond to $W_{4}>W_{8}$. In this region there is a Type 4 periodic solution, and since there are unstable except at the upper extreme of their region of existence (cf I), one expects that the return map will be expanding on at least one interval. This in turn may lead to sensitive dependence on initial conditions in this interval. If we consider the invariant regions of the return maps for parameters in this region we find that we can have one of the three canonical maps shown in Fig. 11. The type D shown in Fig. 11(a) is a type B-like map in the limit as $c_{1} \rightarrow c_{2}$, but the dynamics are quite different when $c_{1} \neq c_{2}$ because the map is expanding between $c_{1}$ and $c_{2}$. The type E map shown in Fig. 11(b) has four pieces. If we order the four pieces by the order of their respective domains, then all pieces are linear except the third one. The absolute values of the slopes of the first and the last pieces are less than one. If $c_{1}=c_{3}$ type E maps 

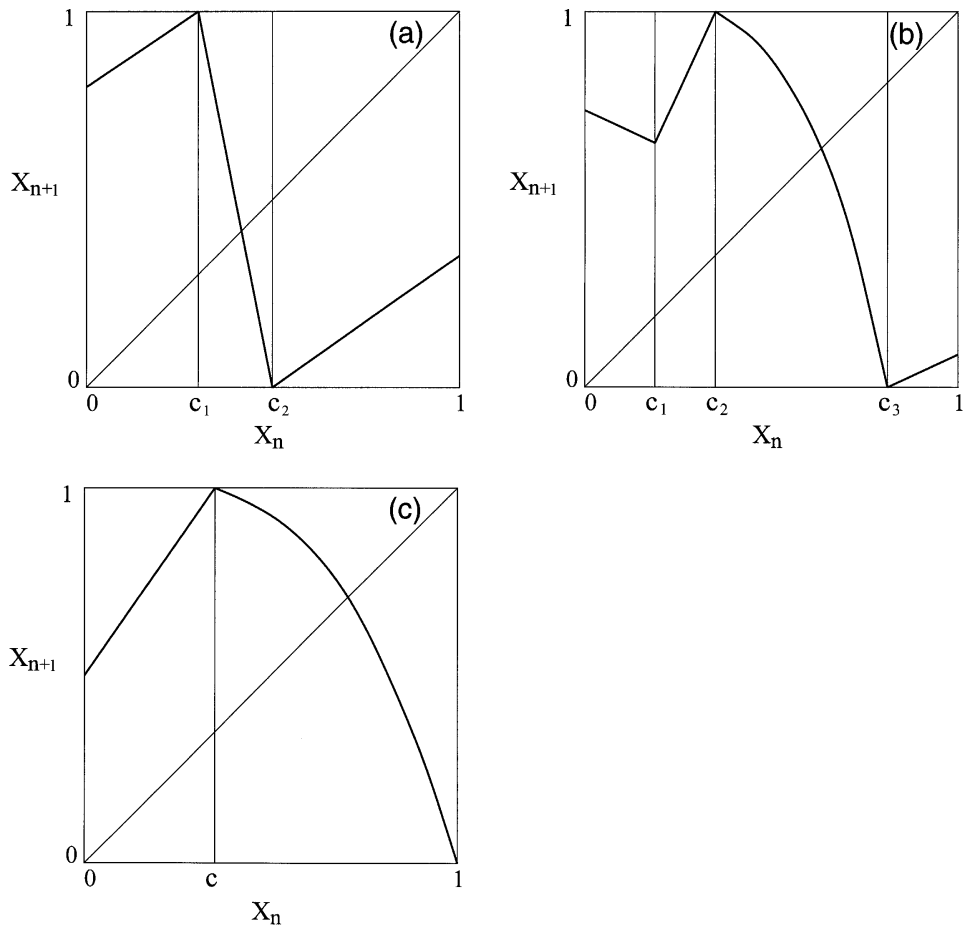

Fig. 11. The new types of canonical maps in Case III. All are continuous and piecewise smooth. (a) A Type D map, which arises at intermediate values of $\theta$. This is the canonical map which derives from the maps in Fig. 2 by restricting them to their minimal invariant set. The graph of the map has three pieces, two of which have positive slope less then 1 , and the other has negative slope with modulus greater than 1 . This type of map reduces to a Type B map when $c_{1}=c_{2}$, but otherwise it has two contracting parts and one expanding part. (b) A type E map, which arises from the restriction of the maps in Fig. 10 to their minimal invariant sets. The graph has four pieces. It is a type A map when $c_{1}=c_{3}$, and a type $\mathrm{F}$ map when $c_{1}=0$ and $c_{3}=1$. (c) A Type F map, which has two branches, one with positive slope (always greater than 1) and the other with negative slope.

reduce to type A maps, while if $c_{1}=0$ and $c_{3}=1$ a type E map reduces to a type F map, which shown in Fig. 11(c). We have not studied the type $\mathrm{D}$ and type $\mathrm{E}$ maps analytically, but we give some numerical results for them in the next section.

\section{Chaotic dynamics}

\section{1. $W_{4}>W_{8}$}

We first consider the dynamics for small fixed $\theta$, and variable $T$ in the transition region between $1: 1$ solution and $2: 1$ solutions. We showed 
in II that for intermediate forcing amplitudes type A maps apply in this region, and we proved that there are only $n: 1$ solutions, and that $n: 1$ solutions and $n+1: 1$ solutions can coexist. That is, there may be bistability and a direct transition between $1: 1$ and 2:1 solutions for small $\theta$ for Case II. However, in Case III the attractor is a periodic orbit when the forcing is on, and there are no type A maps between the boundaries of the $1: 1$ and $2: 1$ regions for small $\theta$. Instead one finds that as $T$ is decreased from the $1: 1$ region the return maps appear in the sequence type $\mathrm{F}$, type $\mathrm{E}$ and type $\mathrm{D}$, and we now investigate what the dynamics are in this region. For this purpose we fix $\theta$ at 0.1 and, using the period as the bifurcation parameter, we iterate the return map from a single point 2000 times and plot the last 100 iterates. We show iterates of the point as a function of the period for $A=1.1$ in Fig. 12. Type $\mathrm{D}$ maps apply for $T$ in the interval $(\sim 0.2, \sim 1.25)$, type $\mathrm{E}$ maps apply in the interval $\sim 1,25, \sim 2.0$ ), and type $\mathrm{F}$ maps apply in the remainder of the locking region $\mathcal{T}_{4}^{1: 1}$. Figure 12 suggests that when $W_{4}>W_{8}$, the character of the attractor goes through the sequence $1: 1 \rightarrow 2: 2 \rightarrow$ irregular $\rightarrow$ period $-5 \rightarrow \cdots \rightarrow$ period $-7 \rightarrow \cdots \rightarrow$ $2: 1 \rightarrow \cdots$ as $T$ decreases from the $1: 1$ region. In particular, one seesthat there may be a direct transition from $2: 2$ to chaos around $T=2.2$, and since the canonical map is of type $\mathrm{F}$ in this region we can prove this analytically.

The type F map is quadratic-like, and one expects similarities with the dynamics and bifurcation sequence for the standard family of quadratic maps $x \rightarrow a x(1-x)$. However the latter are smooth in the parameter $a$, and as a result, the map undergoes a succession of period-doubling bifurcations which lead to chaos (cf. Devaney (1992)).

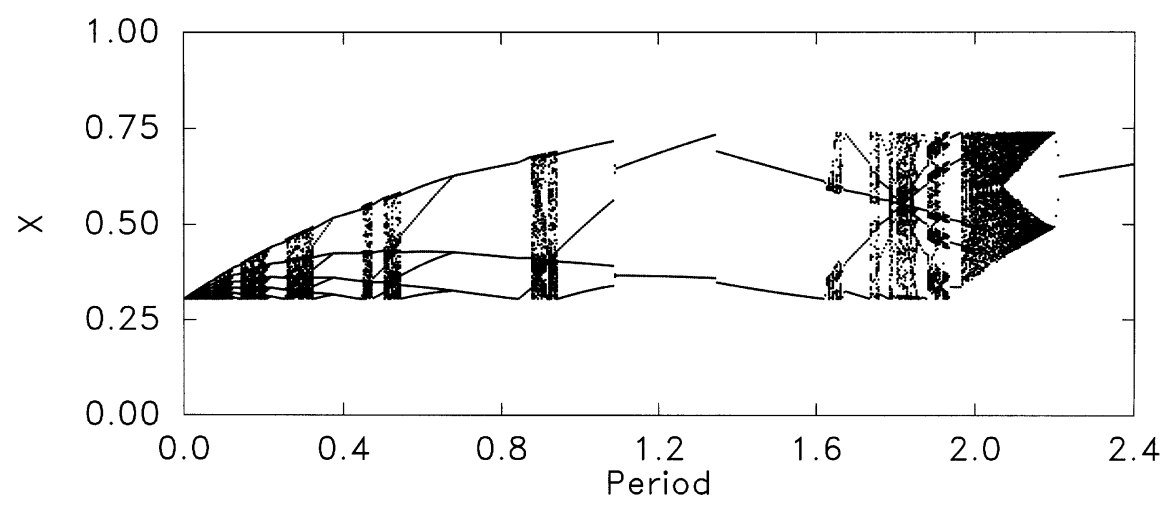

Fig. 12. Images under the return map of the initial point $x_{0}=0.5$ versus the period T for $\theta=0.1$ and $A=1.1$. The initial point is iterated 2000 times and the last 100 iterates are plotted for each period in this figure. 
In contrast, the map shown in Fig. 11(c) is not differentiable at the intersection of the two branches and the slope of the left branch is always greater than 1 . The absolute value of the slope of the right branch of the map increases as the period decreases, and at the intersection of the branches the slope has minimum absolute value $m(A)>0$, and it has maximum absolute value at 1 . The slope of that part of the right branch which is below the fixed point of the first iterate is less than -1 , and there are two fixed point of the second iterate of the return map when $T$ is slightly to the left of the period-doubling bifurcation. At the lower one, the slope of the map is less than -1 and at the upper one (above the fixed point of the first iterate of the map), the slope is greater than -1 . As the period decreases, the upper fixed point reaches the intersection of the two branches and then moves onto the left branch at a critical value $T^{*}$. One can show that below this value of $T$ the absolute values of the slopes of the two branches are greater than 1 . The implication of this fact for the asymptotic dynamics can be understood in terms of the Liapunov exponent, which for a $C^{1}$ map $f$ of an interval is defined by (Collett and Eckmann 1980)

$$
\lambda(x)=\lim _{n \rightarrow \infty} \frac{1}{n} \log \left|\left(f^{n}(x)\right)^{\prime}\right| .
$$

If we restrict attention to orbits that do not pass through the intersection of the branches, the exponent is well defined. It follows that when $T-T^{*}$ is negative and sufficiently small in modulus the Liapunov exponent of the map is positive, and hence the dynamical system has chaotic orbits. We summarize the above discussion in the following proposition.

Proposition 3. Any system whose return map restricted to the invariant interval is a type $F$ map has a direct transition from $2: 2$ phase-locking to chaos after the first period-doubling bifurcation.

At smaller values of $T$ the canonical map is of type $\mathrm{D}$, as noted earlier. The limiting form of these as $c_{2} \rightarrow c_{1}$ is a type B map, and from II we know that the dynamics are nonchaotic for these maps. However for type D maps the central branch has negative slope with absolute value greater than 1, and hence is expanding and may show chaotic behavior. When the parameter $\theta$ and $A$ are fixed and the period $T$ is increased, the slope of the middle branch becomes steeper while the other two pieces become flatter. The bifurcation diagram with $T$ as the bifurcation parameter is embedded in Fig. 12. When $T$ decreases from 1.25 to $\sim 0.2$, the map undergoes a $2: 1 \rightarrow 4: 2$ transition, a transition to irregular dynamics, and then a $3: 2 \rightarrow 3: 1 \rightarrow 6: 2$ transition, a transition to irregular dynamics, and so on. The rotation number is 
not a monotonic function of the parameter $T$, while the rotation number of a type B map, the limiting case of a type D map, is a monotonic function of the parameter. From this bifurcation diagram it appears that the phase-locking regions are separated by intervals of irregular dynamics. Such a sequence has also observed in numerical simulations of the Beeler-Reuter model (Lewis and Guevara 1990).

\section{2. $W_{4}<W_{8}$}

When $W_{4}<W_{8}$, the bifurcation diagram in Fig. 13(a) shows that as the period is decreased the asymptotic dynamics evolve from a pair of $1: 1$ orbits to coexistence of a single $1: 1$ orbit and chaos $\rightarrow$ a single $1: 1$ orbit $\cdots \rightarrow$ period $-4 \rightarrow \cdots \rightarrow$ period $-3 \rightarrow \cdots \rightarrow 2: 1 \cdots \rightarrow$. For much of the domain of $T$, the asymptotic behavior depends on the initial point. For instance, when $T=3.5$ there are two stable $1: 1$ solutions, one near 0.25 and the other near 0.7. The former is a type $21: 1$ solution and the latter a type 41:1 solution. As $T$ decreases the type 4 solution loses stability and first undergoes a period-doubling bifurcation, followed by a direct transition to a chaotic attractor. However the fixed point near 0.25 , which corresponds to a type 2 1:1 solution, persists throughout the first chaotic window. The corresponding return map is as shown in Fig. 13(b). In the chaotic region between $T \sim 3$ and $T \sim 3.2$ there are two invariant regions for this return map: on one there is a stable fixed point which is near 0.25 and on the other the map
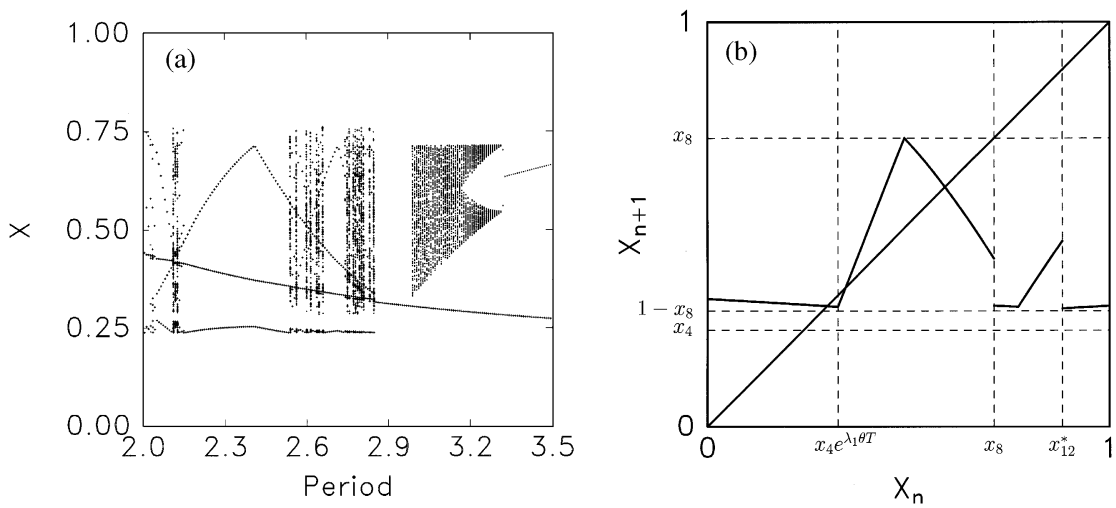

Fig. 13. (a) The bifurcation diagram for $\mathrm{A}=0.9, \theta=0.1$ and $T$ the bifurcation parameter. When $\mathrm{T}$ varies from 3.5 to $=3.0$, the return map has the same structure as the one shown in (b). (b) The return map for $A=0.9, \theta=0.1$, and $T=3.1$. The domain can be divided into two invariant regions: in one there is a stable fixed point which is near $x=0.25$ and the map restricted to the other is a type $F$ map. 
is a type F map. By proposition 3, the dynamical system restricted to the latter invariant set is chaotic.

Thus there is coexistence of a stable $1: 1$ response and a chaotic attractor for a certain range of the forcing period $T$. This coexistence occurs in the small region in $(\theta, T)$ space near the boundary of the type 2 harmonic locking region, where type 2 and type 4 solutions coexist but the latter have lost stability. In contrast, when $W_{4}>W_{8}$ and the period is decreased, the type 4 solutions are stable even when the parameters are outside the type 2 harmonic locking region. In this case the canonical return map does not exhibit bistability of the two different harmonic solutions, and has only type 4 stable harmonic solutions until the first period-doubling bifurcation is reached, after which it goes to chaotic dynamics directly from the $2: 2$ locked solution, as shown in Fig. 12.

\section{Discussion}

Periodically-forced dynamical systems, whether oscillatory or merely excitable in the absence of forcing, arise in a variety of physiological contexts, including cardiac dynamics, neural networks, secretory systems and ciracdian rhythms. There is an enormous literature on forced oscillatory systems, and a well-developed theory of how such systems respond to periodic stimuli exists (Levi 1981, Guckenheimer and Holmes 1983, Glass 1997). The picture is much less complete for forced excitable systems, but understanding such systems is important for understanding a variety of phenomena, most notably in the cardiac context. One of the earliest examples of nontrivial dynamics in a forced excitable system, namely hysteresis, was found by Mines (1913) in periodically-stimulated ventricular tissue. In modern terminology, Mines showed the coexistence of periodic solutions of different rotation number connected via a hysteresis curve (cf. also (Lorente and Davidenko 1990)). More recently experimental studies of periodic forcing of ventricular tissue, Purkinje fibers, or AV node tissue have shown a variety of phase-locking patterns, as well as chaos, and the transitions between different dynamic regimes when the frequency of the forcing is varied have been mapped out in some systems (Chialvo and Jalife 1987b, Jalife 1990, Guevara 1991).

Most theoretical studies of phase-locking patterns in cardiac or neural tissue have relied either on numerical simulation of models of Hodgkin-Huxley type, modified by the addition of other currents (Holden 1976, Guttman et al. 1980, Aihara et al. 1984, Markevich and Sel'kov 1989, Anumonwo et al. 1991), or an experimentally-derived 
interval or circle maps (Vinet et al. 1990, Cheng and Levy 1990, Chialvo 1990b, Lewis and Guevara 1990). In this paper and several preceding papers (Othmer and Watanabe 1994, Xie et al. 1996) we have studied the singular system associated with a piecewise linear (PL) model of an excitable system under periodic step-function forcing. Our results on the coexistence of stable periodic solutions in Type A and C maps (Xie et al. 1996) provide a possible explanation of the hysteresis phenomena observed by Mines (1913) in periodically-stimulated ventricular tissue. The persistence result proved there implies that 'stable' results for the singular system, in a sense made precise there, persist for $\varepsilon>0$ and sufficiently small. Although the piecewise-linear system is not directly related to ionic models of tissue, similar results will obtain for smooth vectorfields of the Fitzhugh-Nagumo type, and it is known that the Hodgkin-Huxley equations can be reduced to the FitzhughNagumo form.

In this paper we restricted attention to sufficiently large amplitude forcing such that the attractor of the flow is a fixed point when the forcing is off and is a periodic orbit when it is on. Many physiological models of excitable systems exhibit periodic oscillations when forced by a steady current, and when forced by a periodically-varying current they show phase-locking. Thus the results given here for the PL model are applicable in that context. One can see from Fig. 8(b) that for a short stimulus (i.e., $\theta$ large), there are only harmonic solutions if $Q_{4}$ is above $Q_{8}$ (cf. Fig. 1). However, under a long stimulus (i.e., $\theta$ small), there are different phenomena in some regions of the parameter space $(\theta, T)$. For instance, there is no direct transition between $1: 1$ and $2: 1$ solutions, but instead stable $2: 2$ and $2: 1$ solutions coexist. Also we have shown that there is a region of the $(\theta, T)$ parameter space between the $1: 1$ and $2: 1$ solutions regions in which the system is chaotic and the transition from $1: 1$ to chaos is a direct transition from the first period-doubling $2: 2$ to chaos. This is in contrast to the usual perioddoubling route to chaos for a smooth quadratic interval map. Lewis and Guevara (1990) found this the same phenomenon, i.e. direct transitions from the first period-doubling to chaos, in the BeelerReuter model (Beeler and Reuter 1977).

We have identified three new canonical return maps that arise under different parameter combinations and have analyzed the behavior of one of them analytically. The type F map is a quadratic-like map that is piecewise smooth and has two branches. One piece has slope greater than one and the other has monotonically increasing absolute value of the slope as the forcing frequency increases, which leads to an unusual bifurcation sequence in which there is a direct transition from the first period-doubling $(2: 2)$ to chaos. This map arises from the 
singular limit of the flow (i.e., for $\varepsilon=0$ ), but for small $\varepsilon>0$, the return map is a smooth, almost 1D map and one expects that the usual period-doubling cascade to chaos will arise. We have also found a significant dependence on the amplitude of the forcing, as shown by the results for $Q_{4}<Q_{8}$ compared with those for $Q_{4}>Q_{8}$ (see Figs. 12 and 13). When $Q_{4}$ is below $Q_{8}$ in Case III, there may be coexistence of chaotic dynamics and stable harmonic phase-locking, depending on the initial conditions, as has been found in circle maps (Glass 1991).

As noted earlier, the problems we address here have been studied by others using ad hoc or experimentally-derived maps ((Chialvo and Jalife 1990, Chialvo et al. 1990, Vinet et al. 1990, Lewis and Geuvara 1990) and references given earlier) in the context of cardiac tissue. These analyses typically use one or more phenomenological characteristics of an action potential, such as the action potential duration, the diastolic interval, and the latency, and maps which relate these quantities in successive periods are determined from fits of experimental data. Since they cannot be derived directly from membrane models, they must rely on experimental fits, but we can determine these quantities from the singular flow as follows. Suppose we consider type 3 solutions and restrict attention to those with a single action potential in each cycle; other cases can be treated in a similar manner. One can determine the stimulation time $\left(T_{\text {stim }}\right)$ directly, and one can compute the action potential duration $(A P D)$, the diastolic interval (i.e., the recovery time) $D I$, and the threshold current for excitation $(T h)$. The relationships among them as follows (Xie et al. 1996).

$$
\begin{aligned}
T_{\text {stim }} & =T_{3}^{+}\left(w_{1}, w_{2}\right) \\
D I & =T_{1}^{-}\left(W_{8}, w_{1}\right) \\
T h & =A_{1}+W_{8} e^{-T_{1}^{-}} \\
A P D & =T_{3}^{-}\left(w_{2}, W_{8}\right)+T_{3}^{+}\left(w_{1}, w_{2}\right) \\
T & =D I+A P D \\
T_{3}^{-}\left(w_{2}, W_{8}\right) & =\frac{1}{\lambda_{3}} \ln \frac{w_{2}-W_{12}}{W_{8}-W_{12}}
\end{aligned}
$$

Since $W_{12}$ is a function of $A, A P D$ is a function of $A, T_{\text {stim }}$, and $D I$. Hence we have

$$
\begin{aligned}
D I_{n} & =T-A P D_{n} \\
A P D_{n+1} & =T_{\text {stim }}+\frac{1}{\lambda_{3}} \ln \frac{W_{11}+\left(W_{8} e^{-D I_{n}}-W_{11}\right) e^{-\lambda_{3} T_{s t i m}}-W_{12}}{W_{8}-W_{12}} \\
T h_{n+1} & =A_{1}+W_{8} e^{-D I_{n}}
\end{aligned}
$$


In this case there is a single branch of the APD curve, but in other cases there may be more. In any case, the resulting discrete dynamical system can be used to connect our results for the flow with the results that others derived from the phenomenological maps.

Several open problems remain. A very important one is to tie the results of either experimentally-determined maps or those from simple low-dimensional flows more closely to the behavior of realistic membrane models for cardiac tissue, such as the Luo-Rudy models. From our results and those of others we have a qualitative understanding of the effects of changes in the frequency and amplitude on the phaselocking patterns, but this still leaves a large gap in that we cannot predict how changes in particular currents will influence the dynamics in more than a very qualitative way. A second problem is to establish a deeper connection between the behavior of forced oscillators and forced excitable systems. Once the stimulus amplitude exceeds the threshold, their behaviors are qualitatively similar. In Alexander et al. (1989b) and Alexander et al. (1990) a theory of forced excitable systems was developed that parallels the classical theory for forced oscillators to a large extent. As was shown there, it is possible to define a rotation number for a certain flow on a two-torus which results from a singular limit of a Fitzhugh-Nagumo type system, despite the fact that the vector field in the singular system is not continuous and orbits are not unique. In fact the associated circle map is set-valued. In the theory developed in Alexander et al. (1990), either the forcing had to appear in the equations for the slow variables or it had to vary slowly enough so that it could be transferred to the slow variables, in a sense made precise there. However, the theory does not apply when the forcing is a step function in time applied to the 'fast' or 'voltage-like' variable, which is the case studied here, and it remains to establish a closer connection in that case.

\section{Appendix: Stability of subharmonic solutions}

In I we have studied the stability of harmonic solutions. Type 1 1:1 solutions are stable whenever they exist and type 5 and type $61: 1$ solutions are unstable whenever they exist. However, type 2 and type 3 solutions are stable for the parameters in the most of the regions where they exist and unstable in the small part of the regions. In contrast type 4 solutions are unstable in most of the region where they exist and stable in the small part of the region. As we stated in I subthreshold solutions are always asymptotically stable. 
In II we have studied subharmonic solutions under medium amplitude stimulus (i.e., $A \in\left(A_{1}, A_{2}\right)$ ), where we showed that there exist three types of $2: 1$ solutions (type 1 , type 2 , and type 3 ), and in sect. 3 we found six different types of $2: 1$ solutions under large amplitude stimulus. In this section we study the stability of the subharmonic solutions. The stability of subharmonic solutions with period $n$ is determined by the derivative of the $n^{\text {th }}$ iterate return map at the fix points. For instance a 2:1 subharmonic solution is stable if the absolute value of the derivative of the second iterate return map is less than one and unstable if it is great than one. In the following we construct the second iterate of the return map for each type of $2: 1$ solutions and study it analytically. We show the regions in which the stable $2: 1$ solutions exist in the parameter space.

1. Type 1 solutions. For a type 1 solution, the second iterate of the return map is

where

$$
\phi_{1}(w)=W_{11}+\left(w_{02} e^{-\lambda_{1} \theta T}-W_{11}\right) e^{-\lambda_{3}(1-\theta) T},
$$

Thus,

$$
w_{02}=W_{5}+\left(w e^{-\lambda_{1} \theta T}-W_{5}\right) e^{-\lambda_{1}(1-\theta) T}
$$

$$
0<\phi_{1}^{\prime}(w)=e^{-\lambda_{1}(1+\theta) T-\lambda_{3}{ }^{(1-\theta) T}}<1
$$

for all $w$. Therefore, type 1 solutions are asympotically stable whenever they exist.

2. Type 2 solutions. For a type 2 solution, the second iterate of the return map is

$$
\phi_{2}(w)=W_{5}+\left(W_{10}-W_{5}\right) e^{-\lambda_{1}(1-\theta) T}\left(\frac{W_{11}-w_{02} e^{-\lambda_{1} \theta T}}{W_{11}-W_{10}}\right)^{\lambda_{1} / \lambda_{3}},
$$

where $w_{02}=W_{5}+\left(w e^{-\lambda_{1} \theta T}-W_{5}\right) e^{-\lambda_{1}(1-\theta) T}$

Hence, at the fixed point (w),

$$
\phi_{2}^{\prime}(w)=-\left(\frac{\lambda_{1}}{\lambda_{3}}\right)\left(\frac{w-W_{5}}{W_{11}-w_{02} e^{-\lambda_{1} \theta T}}\right) e^{-\lambda_{1}(1+\theta) T}<0 .
$$

since $w>W_{5}$ and $W_{11}>w_{02} e^{-\lambda_{1} \theta T}$ for a type 2 solution. Thus, if $\phi_{2}^{\prime}(w)<-1$, the solution is unstable and if $\phi_{2}^{\prime}(w)>-1$, the solution is stable.

If $\phi_{2}^{\prime}(w)<-1$, then

$$
\begin{gathered}
\lambda_{1}\left(w-W_{5}\right)>\lambda_{3}\left\{W_{11}-\left[W_{5}+\left(w e^{-\lambda_{1} \theta T}-W_{5}\right) e^{-\lambda_{1}(1-\theta) T}\right]\right. \\
\left.\times e^{-\lambda_{1} \theta T}\right\} e^{\lambda_{1}(1+\theta) T}
\end{gathered}
$$


and then,

$$
\left(\lambda_{1}+\lambda_{3}\right) w>\lambda_{3} W_{11} e^{\lambda_{1}(1+\theta) T}+W_{5}\left(\lambda_{1}+\lambda_{3} e^{\lambda_{1} \theta T}-\lambda_{3} e^{\lambda_{1} T}\right)
$$

Let $B_{2}=\left(1 /\left(\lambda_{1}+\lambda_{3}\right)\right)\left(\lambda_{3} W_{11} e^{\lambda_{1}(1+\theta) T}+W_{5}\left(\lambda_{1}+\lambda_{3} e^{\lambda_{1} \theta T}-e^{\lambda_{1} T}\right)\right)$. Hence, if $w>B_{2}$, then $\phi_{2}^{\prime}(w)<-1$, i.e., the solution is unstable. And if $w<B_{2}$, then $\phi_{2}^{\prime}(w)>-1$, i.e., the solution is stable.

3. Type 3 solutions. For a type 3 solution, the second iterate of the return map is

$$
\phi_{3}(w)=W_{11}+\left(w_{02} e^{-\lambda_{1} \theta T}-W_{11}\right) e^{-\lambda_{3}(1-\theta) T},
$$

where $w_{02}=W_{5}+\left(w_{01}-W_{5}\right) e^{-\lambda_{1}(1-\theta) T}$ and $w_{01}$

$$
=W_{8} e^{-\lambda_{1} \theta T}\left(\frac{W_{12}-w}{W_{12}-W_{8}}\right)^{\lambda_{1} / \lambda_{3}}
$$

Hence, at the fixed point (w),

$$
\phi_{3}^{\prime}(w)=-\left(\frac{\lambda_{1}}{\lambda_{3}}\right)\left(\frac{w_{01}}{W_{12}-w}\right) e^{-\lambda_{3}(1-\theta) T-\lambda_{1} T}<0 .
$$

since $w_{01}>0$ and $W_{12}>w$ for a type 3 solution. Thus, if $\phi_{3}^{\prime}(w)<-1$, the solution is unstable and if $\phi_{3}^{\prime}(w)>-1$, the solution is stable.

If $\phi_{3}^{\prime}(w)<-1$, then

$$
\begin{gathered}
\lambda_{3}\left(W_{12}-w\right)<\lambda_{1}\left\{W_{5} e^{-\lambda_{3}(1-\theta) T}\left(e^{-\lambda_{1} T}-e^{-\lambda_{1} \theta T}\right)\right. \\
\left.+W_{11}\left(e^{-\lambda_{3}(1-\theta) T}-1\right)+w\right\},
\end{gathered}
$$

and then,

$$
\begin{aligned}
\left(\lambda_{1}+\lambda_{3}\right) w> & \lambda_{3} W_{12}+\lambda_{1} W_{11}\left(1-e^{-\lambda_{3}(1-\theta) T}\right) \\
& +\lambda_{1} W_{5} e^{-\lambda_{3}(1-\theta) T}\left(e^{-\lambda_{1} \theta T}-e^{-\lambda_{1} T}\right)
\end{aligned}
$$

Let

$$
\begin{aligned}
B_{3}= & \left(1 /\left(\lambda_{1}+\lambda_{3}\right)\right)\left(\lambda_{3} W_{12}+\lambda_{1} W_{11}\left(1-e^{\lambda_{3}(1-\theta) T}\right)\right. \\
& \left.+\lambda_{1} W_{5} e^{-\lambda_{3}(1-\theta) T}\left(e^{-\lambda_{1} \theta T}-e^{-\lambda_{1} T}\right)\right) .
\end{aligned}
$$

Hence, if $w>B_{3}$, then $\phi_{3}^{\prime}(w)<-1$, i.e., the solution is unstable. And if $w<B_{3}$, then $\phi_{3}^{\prime}(w)>-1$, i.e., the solution is stable.

4. Type 4 solutions. For a type 4 solution, the second iterate of the return map is

$$
\phi_{4}(w)=W_{11}+\left(W_{4}-W_{11}\right) e^{-\lambda_{3}(1-\theta) T}\left(\frac{w_{02} e^{-\lambda_{1} \theta T}-W_{5}}{W_{4}-W_{5}}\right)^{\lambda / \lambda_{1}},
$$

where $w_{02}=W_{5}+\left(w e^{-\lambda_{1} \theta T}-W_{5}\right) e^{-\lambda_{1}(1-\theta) T}$ 
Hence, at the fixed point (w),

$$
\phi_{4}^{\prime}(w)=-\left(\frac{\lambda_{3}}{\lambda_{1}}\right)\left(\frac{W_{11}-w}{w_{02} e^{-\lambda_{1} \theta T}}-W_{5}\right) e^{-\lambda_{1}(1+\theta) T}<0 .
$$

since $w>W_{11}$ and $W_{5}<w_{02} e^{-\lambda_{1} \theta T}$ for a type 4 solution. Thus, if $\phi_{4}^{\prime}(w)<-1$, the solution is unstable and if $\phi_{4}^{\prime}(w)>-1$, the solution is stable.

$$
\text { If } \phi_{4}^{\prime}(w)<-1 \text {, then }
$$

$$
\lambda_{3}\left(W_{11}-w\right)>\lambda_{1}\left\{W_{5}-\left(e^{-\lambda_{1} T}-e^{-\lambda_{1} \theta T}-e^{-\lambda_{1}(1-\theta) T}\right)+w\right\}
$$

and then,

$$
\left(\lambda_{1}+\lambda_{3}\right) w<\lambda_{3} W_{11}+\lambda_{1} W_{5}\left(e^{\lambda_{1} \theta T}+e^{\lambda_{1}(1+\theta) T}-e^{\lambda_{1} T}\right)
$$

Let

$$
B_{4}=\left(1 /\left(\lambda_{1}+\lambda_{3}\right)\right)\left(\lambda_{3} W_{11}+\lambda_{1} W_{5}\left(e^{\lambda_{1} \theta T}+e^{\lambda_{1}(1+\theta) T}-e^{\lambda_{1} T}\right)\right) .
$$

Hence, if $w<B_{4}$, then $\phi_{4}^{\prime}(w)<-1$, i.e., the solution is unstable. And if $w>B_{4}$, then $\phi_{4}^{\prime}(w)>-1$, i.e., the solution is stable.

5. Type 5 solutions. For a type 5 solution, the second iterate of the return map is

$$
\begin{aligned}
\phi_{5}(w)= & W_{5}+\left(\frac{W_{10}-W_{5}}{W_{4}-W_{5}}\right)\left(\frac{W_{11}-W_{4}}{W_{11}-W_{10}}\right)^{\lambda_{1} / \lambda_{3}} \\
& \times e^{-\lambda_{1}(1-\theta) T}\left(w_{02} e^{-\lambda_{1} \theta T}-W_{5}\right),
\end{aligned}
$$

where $w_{02}=W_{5}+\left(w e^{-\lambda_{1} \theta T}-W_{5}\right) e^{-\lambda_{1}(1-\theta) T}$

Hence, at the fixed point (w),

$$
\begin{aligned}
\phi_{5}^{\prime}(w) & =\left(\frac{W_{10}-W_{5}}{W_{4}-W_{5}}\right)\left(\frac{W_{11}-W_{4}}{W_{11}-W_{10}}\right)^{\lambda_{1} / \lambda_{3}} e^{-2 \lambda_{1} T} \\
& =e^{-\lambda_{1}\left(T_{0}-2 T\right)},
\end{aligned}
$$

where $\quad T_{0}=\left(1 / \lambda_{1}\right)\left(\ln \left(W_{10}-W_{5}\right) /\left(W_{4}-W_{5}\right)\right)+\left(1 / \lambda_{3}\right)\left(\ln \left(W_{11}-W_{4}\right) /\right.$ $\left.\left(W_{11}-W_{10}\right)\right)$ is the time required for each circuit while the forcing remain on. Since $T_{0}>2 T, \phi_{5}^{\prime}(w)>1$. Thus, type 5 solutions are unstable whenever they exist.

6. Type 6 solutions. For a type 6 solution, the second iterate of the return map is

$$
\phi_{6}(w)=W_{11}+\left(W_{4}-W_{11}\right) e^{-\lambda_{3}(1+\theta) T}\left(\frac{w_{02} e^{-\lambda_{1} \theta T}-W_{5}}{W_{4}-W_{5}}\right)^{\lambda_{3} / \lambda_{1}},
$$


where $w_{02}=W_{5}+\left(w_{01}-W_{5}\right) e^{-\lambda_{1}(1-\theta) T}$ and

$$
w_{01}=W_{4} e^{-\lambda_{1} \theta T}\left(\frac{W_{12}-w}{W_{12}-W_{8}}\right)^{\lambda_{1} / \lambda_{3}}
$$

Hence, at the fixed point (w),

$$
\phi_{6}^{\prime}(w)=\left(\frac{W_{11}-w}{W_{12}-w}\right)\left(\frac{w_{01}}{w_{02} e^{-\lambda_{1} \theta T}-W_{5}}\right) e^{-\lambda_{1} T}
$$

Since $\left.T=\left(1 / \lambda_{1}\right) \ln \left(w_{02} / w_{03}\right)\left(w_{01}-W_{5}\right) /\left(w_{02}-W_{5}\right)\right)$ for a type 6 solution, where $w_{03}=w_{02} e^{-\lambda_{1} \theta T}$, it follows that

$$
\begin{aligned}
\phi_{6}^{\prime}(w) & =\left(\frac{W_{11}-w}{W_{12}-w}\right)\left(\frac{w_{01}}{w_{03}-W_{5}}\right)\left(\frac{w_{03}}{w_{02}}\right)\left(\frac{w_{02}-W_{5}}{w_{01}-W_{5}}\right) \\
& >\left(\frac{w_{01}}{w_{02}}\right)\left(\frac{w_{02}-W_{5}}{w_{03}-W_{5}}\right)\left(\frac{w_{03}}{w_{01}-W_{5}}\right) \\
& >\left(\frac{w_{01}}{w_{02}}\right)\left(\frac{w_{02}}{w_{03}}\right)\left(\frac{w_{03}}{w_{01}-W_{5}}\right) \\
& =\left(\frac{w_{01}}{w_{01}-W_{5}}\right)>1 .
\end{aligned}
$$

Therefore, type 6 solutions are unstable whenever they exist.

We summarize the above results in the following Proposition.

Proposition 4. In the parameter space $(\theta, T)$, the following holds for $2: 1$ solutions.

(a) Type 1 solutions are asymptotically stable whenever they exist.

(b) A type $i(i=2$ or 3$)$ is asymptotically stable if the fixed point $w_{i}<B_{i}$ and unstable if $w_{i}>B_{i}$.

(c) A type 4 solution is asymptotically stable if the fixed point $w_{4}>B_{4}$ and unstable if $w_{4}<B_{4}$.

(d) Solutions of types 5 and 6 are unstable whenever they exist.

For our standard parameters, solutions of types 2 and 3 are stable whenever they exist.

\section{References}

Aihara, Kazuyuki, Matsumoto, Gen, and Ikegaya, Yuhji, 1984. Periodic and Nonperiodic Responses of a Periodically Forced Hodgkin-Huxley Oscillator. J. Theor. Biol., 109, 249-269 
Alexander, J. C., Doedel, E. J., and Othmer, H. G. 1989a. Resonance and phase-locking in excitable systems. Pages 1-37 of: Othmer, H. G. (ed), The Dynamics of Excitable Media. Amer. Math. Soc

Alexander, J. C., Doedel, E. J., and Othmer, H. G. 1989b. Resonance and phase-locking in excitable systems. Pages 1-37 of: Othmer, H. G. (ed), The Dynamics of Excitable Media. Amer. Math. Soc

Alexander, J. C., Doedel, E. J., and Othmer, H. G. 1990. On the resonance structure in a forced excitable system. SIAM J. Appl. Math., 50(5), 1373-1418

Anumonwo, J. M. B., Delmar, M., Vinet, A., Michaels, D. C., and Jalife, J. 1991. Phase resetting and entrainment of pacemaker activity in single sinus nodal cells. Circ. Res., 68, 1138-1153

Aronson, D., Doedel, E. J., and Othmer, H. G. 1986. Bistable behavior in coupled oscillators. Pages 221-231 of: Othmer, H. G. (ed), Nonlinear Oscillations in Biology and Chemistry. Lect. Notes in Biomath., vol. 66. Springer-Verlag

Beeler, G. W. and Reuter, H. 1977. Reconstruction of the action potential of ventricular myocardial fibres. J. Physiol., 268, 177-210

Boyland, P. L. 1986. Bifurcations of Circle Maps: Arnol'd Tongues, Bistability and Rotation Intervals. Comm. Math. Phys., 106(3), 353-381

Cheng, J. and Levy, M. 1990. Feedback mechanisms and dynamics of atrioventricular propagation. Ann. N. Y. Acad. Sci., 591, 1-10

Chialvo, D. R. 1990a. Toward very simple generic models of excitable cells. Order and chaos in cardiac tissues. Conjectures and facts. Anns. N. Y. Acad. Sci., 591, 351-366

Chialvo, D. R. 1990b. Toward very simple generic models of excitable cells. Order and chaos in cardiac tissues. Conjectures and facts. Anns. N. Y. Acad. Sci., 591, 351-366

Chialvo, D. R. and Jalife, J. 1987a. Non-linear dynamics of cardiac excitation and impulse propagation. Nature, 330, 749-752

Chialvo, D. R. and Jalife, J. 1987b. Non-linear dynamics of cardiac excitation and impulse propagation. Nature, 330, 749-752

Chialvo, D. R. and Jalife, J. 1990. Cardiac Electrophysiology. W. B. Saunders. Pages 201-214. Chap. On the nonlinear equilibrium of the heart: Locking behavior and chaos in Purkinje fibers, pages 201-214

Chialvo, D. R., Michaels, D. C., and Jalife, J. 1990. Supernormal excitability as a mechanism of chaotic dynamics of activation in cardiac. Purkinje fibers. Circ. R., 66, 525-545

Collet, J. P. and Eckmann, J. P. 1980. Iterated Maps of the Interval as Dynamical Systems. Boston- Birkäuser-Verlag

Devaney, R. L. 1992. A First Course in Chaotic Dynamical Systems: Theory and experiment. 1 edn. Studies in nonlinearity. Reading, Massachusetts: AddisonWesley

Glass, L. 1991. Cardiac arrhythmias and circles maps-a classical problem. Chaos, 1, $13-20$

Glass, L. 1997. The topology of phase resetting and the entrainment of limit cycles, in Case Studies in Mathematical Modeling, H. G. Othmer, et al., eds., Prentice Hall. Pages 255-276. pages 255-276

Glass, L. and Zeng, W. 1990. Complex bifurcations and chaos in simple theoretical models of cardiac oscillation. Pages 316-327 of: Jalife, J. (ed), Mathematical Approaches to Cardiac Arrhythmias. New York, New York: The New York Academy of Science

Glass, Leon, Guevara, Michael R., and Shrier, Alvin. 1987. Universal bifurcations and the classification of cardiac arrhythmias. Ann. NYAS, 504, 168-178 
Guckenheimer, J. and Holmes, P. J. 1983. Nonlinear Oscillations, Dynamical Systems and Bifurcations of Vector Fields. Applied Mathematical Sciences, vol. 42. New York: Springer-Verlag

Guevera, M. R. 1991. Iteration of the human atrioventricular (AV) nodal recovery curve predicts many rhythms of AV block, in Theory of Heart. Springer-Verlag, 313-358

Guevara, M. R., Ward, G., Shrier, A., and Glass, L. 1984. Electrical alternans and period-doubling bifurcations. In: IEEE Computers in Cardiology. 167-170

Guttman, Rita, Feldman, Lance, and Jakobsson, Eric. 1980. Frequency entrainment of squid axon membrane. J. Membrane Biol., 56, 9-18

Holden, A. V. 1976. The response of excitable membrane models to a cyclic input. Biol. Cybernetics, 21, 1-7

Jalife, J. (ed). 1990. Mathematical Approaches to Cardiac Arrhythmias. New York: New York Academy of Sciences

Levi, M. 1981. Qualitative analysis of the periodically forced relaxation oscillators. Mem. Am. Math. Soc., 32(244), 1-147

Lewis, T. and Guevara, M. 1990. Chaotic dynamics in an ionic model of the propagated cardiac action potential. J. Theor. Biol., 146, 407-432.

Lorente, P. and Davidenko, J. 1990. Mathematical Approaches to Cardiac Arrhythmias. New York Academy of Sciences. Chap. Hysteresis phenomena in excitable tissues.

Markevich, N. I. and Sel'kov, E. E. 1989. Parametric resonance and amplification in excitable membranes. The Hodgkin-Huxley model. J. Theor. Biol., 140, 27-38.

Mines, G. R. 1913. On the dynamic equilibrium in the heart. J. Physiol., 46, 349-383

Othmer, Hans G. and Watanabe, Masaji. 1994. Resonances in excitable systems under step function forcing. I Harmonic solutions. Advs. in Math. and Applics., 4, 399-441

Rinzel, J. and Troy, C. W. 1983. A one-variable map analysis of bursting in the Belousov-Zhabotinskii Reaction. Contemporary Mathematics, 17, 411-427

Sturis, J., Cauter, E. Van, Blackman, J. D., and Polonsky, K. S. 1991. Entrainment of pulsatile insulin secretion by oscillatory glucose infusion. J. Clin. Invest., 87, 439-445

Vinet, A., Chialvo, D. R., Michaels, D. C., and Jalife, J. 1990. Nonlinear dynamics of rate-dependent activation in models of single cardia cells. Circ. Res., 67, 1510-1524

Wetsel, W. C., Valenca, M. M., Mercenthaler, I., Liposits, Z., Lopez, F. J., Weiner, R. I., Mellon, P., and Negro-Villar, A. 1992. Intrinsic pulsatile secretory activity of immortalized lutenizing hormone-releasing hormone-secreting heurons. PNAS, 89(May), 4149-4153

Xie, M., Othmer, H. G., and Watanabe, M. 1996. Resonance in excitable systems under step-function forcing. II. Subharmonic solutions and persistence. Physica D, 98, $75-110$ 